\title{
Non-squareness properties of Orlicz-Lorentz function spaces
}

\author{
Paweł Foralewski ${ }^{*}$, Henryk Hudzik ${ }^{1}$ and Paweł Kolwicz ${ }^{2}$
}

"Correspondence:
katon@amu.edu.pl
${ }^{1}$ Faculty of Mathematics and
Computer Science, Adam
Mickiewicz University, Umultowska
87, Poznań, 61-614, Poland
Full list of author information is
available at the end of the article

available at the end of the article

\begin{abstract}
In this paper, criteria for non-squareness and uniform non-squareness of Orlicz-Lorentz function spaces $\Lambda_{\varphi, \omega}$ are given. Since degenerated Orlicz functions $\varphi$ and degenerated weight functions $\omega$ are also admitted, this investigation concerns the most possible wide class of Orlicz-Lorentz function spaces.

It is worth recalling that uniform non-squareness is an important property, because it implies super-reflexivity as well as the fixed point property (see James in Ann. Math. 80:542-550, 1964; Pacific J. Math. 41:409-419, 1972 and García-Falset et al. in J. Funct. Anal. 233:494-514, 2006).

MSC: 46B20; 46B42; 46A80; 46E30
\end{abstract}

Keywords: uniform non-squareness; non-squareness; Orlicz-Lorentz space; Lorentz space; Orlicz function; Luxemburg norm; strict monotonicity; uniform monotonicity; reflexivity; super-reflexivity; fixed point property

Uniform non-squareness of Banach spaces has been defined by James as the geometric property which implies super-reflexivity (see $[1,2])$. So, after proving this property for a Banach space, we know, without any characterization of the dual space, that it is superreflexive, so reflexive as well. Recently, García-Falset, Llorens-Fuster and MazcuñanNavarro have shown that uniformly non-square Banach spaces have the fixed point property (see [3]).

Therefore, it was natural and interesting to look for criteria of non-squareness properties in various well-known classes of Banach spaces. Among a great number of papers concerning this topic, we list here [4-12].

The problem of uniform non-squareness of Calderón-Lozanovskii spaces was initiated by Cerdà, Hudzik and Mastyło in [13]. Since the class of Orlicz-Lorentz spaces is a subclass of Calderón-Lozanovskii spaces, we can say that also the problem of uniform nonsquareness of Orlicz-Lorentz spaces was initiated in [13]. However, the results of our paper show that those results were only some sufficient conditions for uniform non-squareness which were very far from being necessary and sufficient. Analogous results for OrliczLorentz sequence spaces were presented in [14], but the techniques of the proofs in the function case are different (in some parts completely different) than in the sequence case.

\section{Preliminaries}

We say that a Banach space $(X,\|\cdot\|)$ is non-square if $\min \left(\left\|\frac{x-y}{2}\right\|,\left\|\frac{x+y}{2}\right\|\right)<1$ for any $x$ and $y$ from $S(X)$ (the unit sphere of $X$ ). A Banach space $X$ is said to be uniformly non-square if 
there exists $\delta \in(0,1)$ such that $\min \left(\left\|\frac{x-y}{2}\right\|,\left\|\frac{x+y}{2}\right\|\right) \leq 1-\delta$ for any $x, y \in B(X)$ (the unit ball of $X)$. In the last definition, the unit ball $B(X)$ can be replaced, equivalently, by the unit sphere $S(X)$.

Let $L^{0}=L^{0}([0, \gamma))$ be the space of all (equivalence classes of) Lebesgue measurable realvalued functions defined on the interval $[0, \gamma)$, where $\gamma \leq \infty$. For any $x, y \in L^{0}$, we write $x \leq y$ if $x(t) \leq y(t)$ almost everywhere with respect to the Lebesgue measure $m$ on the interval $[0, \gamma)$.

Given any $x \in L^{0}$, we define its distribution function $\mu_{x}:[0,+\infty) \rightarrow[0, \gamma]$ by

$$
\mu_{x}(\lambda)=m(\{t \in[0, \gamma):|x(t)|>\lambda\})
$$

(see $[15,16]$ and $[17])$ and the non-increasing rearrangement $x^{*}:[0, \gamma) \rightarrow[0, \infty]$ of $x$ as

$$
x^{*}(t)=\inf \left\{\lambda \geq 0: \mu_{x}(\lambda) \leq t\right\}
$$

(under the convention $\inf \emptyset=\infty$ ). We say that two functions $x, y \in L^{0}$ are equimeasurable if $\mu_{x}(\lambda)=\mu_{y}(\lambda)$ for all $\lambda \geq 0$. Then we obviously have $x^{*}=y^{*}$.

Let $\left(R_{1}, \Sigma_{1}, \mu_{1}\right)$ and $\left(R_{2}, \Sigma_{2}, \mu_{2}\right)$ be totally $\sigma$-finite measure spaces. A map $\sigma$ from $R_{1}$ into $R_{2}$ is called a measure preserving transformation if for each $\Sigma_{2}$-measurable subset $A$ from $R_{2}$, the set $\sigma^{-1}(A)=\left\{t \in R_{1}: \sigma(t) \in A\right\}$ is a $\Sigma_{1}$-measurable subset of $R_{1}$ and $\mu_{1}\left(\sigma^{-1}(A)\right)=\mu_{2}(A)$ (see [15]). It is well known that a measure preserving transformation induces equimeasurability, that is, if $\sigma$ is a measure preserving transformation, then $x$ and $x \circ \sigma$ are equimeasurable functions. The converse is false (see [15]).

A Banach space $E=(E, \leq,\|\cdot\|)$, where $E \subset L^{0}$, is said to be a Köthe space if the following conditions are satisfied:

(i) if $x \in E, y \in L^{0}$ and $|y| \leq|x|$, then $y \in E$ and $\|y\| \leq\|x\|$,

(ii) there exists a function $x$ in $E$ that is strictly positive on the whole $[0, \gamma)$.

Recall that a Köthe space $E$ is called a symmetric space if $E$ is rearrangement invariant which means that if $x \in E, y \in L^{0}$ and $x^{*}=y^{*}$, then $y \in E$ and $\|x\|=\|y\|$ (see [18]). For basic properties of symmetric spaces, we refer to $[15,16]$ and [17].

In the whole paper, $\varphi$ denotes an Orlicz function (see [19-21]), that is, $\varphi:[-\infty, \infty] \rightarrow$ $[0, \infty]$ (our definition is extended from $R$ into $R^{e}$ by assuming $\varphi(-\infty)=\varphi(\infty)=\infty$ ) and $\varphi$ is convex, even, vanishing and continuous at zero, left continuous on $(0, \infty)$ and not identically equal to zero on $(-\infty, \infty)$. Let us denote

$$
\begin{aligned}
& a_{\varphi}=\sup \{u \geq 0: \varphi(u)=0\}, \\
& b_{\varphi}=\sup \{u \geq 0: \varphi(u)<\infty\}
\end{aligned}
$$

and

$$
\delta=\sup \left\{u \geq 0: \varphi\left(\frac{u}{2}\right)=\frac{1}{2} \varphi(u)\right\} .
$$

Let us note that if $a_{\varphi}>0$, then $\delta=a_{\varphi}$, while left continuity of $\varphi$ on $(0, \infty)$ is equivalent to the fact that $\lim _{u \rightarrow\left(b_{\varphi}\right)^{-}} \varphi(u)=\varphi\left(b_{\varphi}\right)$. 
Recall that an Orlicz function $\varphi$ satisfies the condition $\Delta_{2}$ for all $u \in \mathbb{R}\left(\varphi \in \Delta_{2}(\mathbb{R})\right.$ for short) if there exists a constant $K>0$ such that the inequality

$$
\varphi(2 u) \leq K \varphi(u)
$$

holds for any $u \in \mathbb{R}$ (then we have $a_{\varphi}=0$ and $b_{\varphi}=\infty$ ). Analogously, we say that an Orlicz function $\varphi$ satisfies the condition $\Delta_{2}$ at infinity $\left(\varphi \in \Delta_{2}(\infty)\right.$ for short) if there exist a constant $K>0$ and a constant $u_{0} \geq 0$ such that $\varphi\left(u_{0}\right)<\infty$ and inequality (1) holds for any $u \geq u_{0}$ (then we obtain $b_{\varphi}=\infty$ ).

For any Orlicz function $\varphi$, we define its complementary function in the sense of Young by the formula

$$
\psi(u)=\sup _{v>0}\{|u| v-\varphi(v)\}
$$

for all $u \in \mathbb{R}$. It is easy to show that $\psi$ is also an Orlicz function.

Let $\omega:[0, \gamma) \rightarrow \mathbb{R}_{+}$be a non-increasing and locally integrable function called a weight function. Let us define

$$
\begin{aligned}
& \gamma_{0}=\sup \{t \geq 0: \omega \text { is constant on }(0, t)\} \\
& \alpha=\sup \{t \geq 0: \omega(t)>0\}
\end{aligned}
$$

We say that a weight function $\omega$ is regular if there exists $\eta>0$ such that

$$
\int_{0}^{2 t} \omega(t) d t \geq(1+\eta) \int_{0}^{t} \omega(t) d t
$$

for any $t \in[0, \gamma / 2)$ (see [22-25]). Note that if the weight function $\omega$ is regular, then $\int_{0}^{\infty} \omega(t) d t=\infty$ in the case when $\gamma=\infty$ and $\alpha>\gamma / 2$ whenever $\gamma<\infty$.

Now we recall the definition of Orlicz-Lorentz spaces. These spaces were introduced by Kamińska (see [26, 27] and [24]) at the beginning of 1990s. Her investigations gave an impulse to further investigations of the spaces, results of which have been published, among others, in the papers [14, 28-42].

Given any Orlicz function $\varphi$ and a weight function $\omega$, we define on $L^{0}$ the convex modular

$$
I_{\varphi, \omega}(x)=\int_{0}^{\gamma} \varphi\left(x^{*}(t)\right) \omega(t) d t
$$

(see [26] and [28]) and the Orlicz-Lorentz function space

$$
\Lambda_{\varphi, \omega}=\Lambda_{\varphi, \omega}([0, \gamma))=\left\{x \in L^{0}: I_{\varphi, \omega}(\lambda x)<\infty \text { for some } \lambda>0\right\}
$$

(see [26] and [28]), which becomes a Banach symmetric space under the Luxemburg norm

$$
\|x\|=\inf \left\{\lambda>0: I_{\varphi, \omega}(x / \lambda) \leq 1\right\} .
$$


In our investigations, we apply the results concerning the monotonicity properties of Lorentz function spaces that were presented in $[25,43,44]$. Let us recall that the Lorentz function spaces $\Lambda_{\omega}$ (see $\left.[10,18,22,23,45-50]\right)$ are defined by the formula

$$
\Lambda_{\omega}=\Lambda_{\omega}([0, \gamma))=\left\{x \in L^{0}:\|x\|_{\omega}=\int_{0}^{\gamma} x^{*}(t) \omega(t) d t<\infty\right\} .
$$

A Banach lattice $E=(E, \leq,\|\cdot\|)$ is said to be strictly monotone if $x, y \in E, 0 \leq y \leq x$ and $y \neq x$ imply that $\|y\|<\|x\|$. We say that $E$ is uniformly monotone if for any $\varepsilon \in(0,1)$, there is $\delta(\varepsilon) \in(0,1)$ such that $\|x-y\| \leq 1-\delta(\varepsilon)$ whenever $x, y \in E, 0 \leq y \leq x,\|x\|=1$ and $\|y\| \geq \varepsilon$ (see [51]). Recall (see [52]) that in Banach lattices $E$, strict monotonicity and uniform monotonicity are restrictions of rotundity and uniform rotundity (respectively) to couples of comparable elements in the positive cone $E_{+}$only.

Theorem 1.1 ([25], Theorem 2 and [43], Lemma 3.1) The Lorentz function space $\Lambda_{\omega}$ is strictly monotone if and only if $\omega$ is positive on $[0, \gamma)$ and $\int_{0}^{\gamma} \omega(t) d t=\infty$ whenever $\gamma=\infty$.

The following theorem has been proved in [25, Theorem 1] for $\gamma=\infty$. Moreover, applying some ideas from the proof of Theorem 3.7 (see Case 2 on p.2722) in [53], this theorem can be also shown for $\gamma<\infty$.

Theorem 1.2 The Lorentz function space $\Lambda_{\omega}$ is uniformly monotone if and only if the weight function $\omega$ is regular and $\omega$ is positive on $[0, \gamma)$ whenever $\gamma<\infty$.

In our further investigations, we will also apply Lemma 1.1 and Remark 1.1. By convexity of the modular $I_{\varphi, \omega}$, Lemma 1.1 can be proved analogously as in the case of Orlicz spaces ( $c f$. also [43] for considering a more general case).

Lemma 1.1 Suppose that the Orlicz function $\varphi$ satisfies a suitable condition $\Delta_{2}$, that is, $\varphi \in \Delta(\mathbb{R})$ if $\gamma=\infty$ and $\int_{0}^{\infty} \omega(t) d t=\infty$, and $\varphi \in \Delta(\infty)$ otherwise. Then, for any $\varepsilon \in(0,1)$, there exists $\delta=\delta(\varepsilon) \in(0,1)$ such that $\|x\| \leq 1-\delta$ for any $x \in \Lambda_{\varphi, \omega}$ whenever $I_{\varphi, \omega}(x) \leq 1-\varepsilon$. In particular, for any $x \in \Lambda_{\varphi, \omega}$, we then have that $\|x\|=1$ if and only if $I_{\varphi, \omega}(x)=1$.

Remark 1.1 Let $x, y \in \Lambda_{\varphi, \omega}$ and $t \in(0, \gamma)$ be such that $\left(\frac{x+y}{2}\right)^{*}(t)>\lim _{s \rightarrow \infty}\left(\frac{x+y}{2}\right)^{*}(s)=$ $\left(\frac{x+y}{2}\right)^{*}(\infty)$. By [16, Property $7^{\circ}$, p.64], there exists a set $e_{t}=e_{t}\left(\frac{x+y}{2}\right)$ such that $m\left(e_{t}\right)=t$ and

$$
\int_{0}^{t}\left(\frac{x+y}{2}\right)^{*}(s) d s=\int_{e_{t}}\left|\frac{x+y}{2}\right|(s) d s
$$

Defining $t(x)=m\left(\operatorname{supp} x \cap e_{t}\right)$ and $t(y)=m\left(\operatorname{supp} y \cap e_{t}\right)$, by convexity of the modular $I_{\varphi, \omega}$, we have

$$
\begin{aligned}
\int_{0}^{t} \varphi\left(\left(\frac{x+y}{2}\right)^{*}(s)\right) \omega(s) d s= & I_{\varphi, \omega}\left(\left(\frac{x+y}{2}\right) \chi_{e_{t}}\right) \leq \frac{1}{2} I_{\varphi, \omega}\left(x \chi_{e_{t}}\right)+\frac{1}{2} I_{\varphi, \omega}\left(y \chi_{e_{t}}\right) \\
= & \frac{1}{2} \int_{0}^{t(x)} \varphi\left(\left(x \chi_{e_{t}}\right)^{*}(s)\right) \omega(s) d s \\
& +\frac{1}{2} \int_{0}^{t(y)} \varphi\left(\left(y \chi_{e_{t}}\right)^{*}(s)\right) \omega(s) d s .
\end{aligned}
$$


Denoting $A_{t}=[0, \gamma) \backslash e_{t}, a(x)=m\left(\operatorname{supp} x \cap A_{t}\right), a(y)=m\left(\operatorname{supp} y \cap A_{t}\right)$ and applying convexity of the modular $I_{\varphi, t}$, defined by the formula

$$
I_{\varphi, t}(x)=\int_{0}^{\gamma} \varphi\left(x^{*}(s)\right) \omega(t+s) d s
$$

(if $\gamma<\infty$, we assume that $\omega(t+s)=0$ for $s \geq \gamma-t$ ), we get

$$
\begin{aligned}
\int_{t}^{\gamma} \varphi\left(\left(\frac{x+y}{2}\right)^{*}(s)\right) \omega(s) d s= & \int_{0}^{\gamma} \varphi\left(\left(\left(\frac{x+y}{2}\right) \chi_{A_{t}}\right)^{*}(s)\right) \omega(t+s) d s \\
= & I_{\varphi, t}\left(\left(\frac{x+y}{2}\right) \chi_{A_{t}}\right) \leq \frac{1}{2} I_{\varphi, t}\left(x \chi_{A_{t}}\right)+\frac{1}{2} I_{\varphi, t}\left(y \chi_{A_{t}}\right) \\
= & \frac{1}{2} \int_{0}^{a(x)} \varphi\left(\left(x \chi_{A_{t}}\right)^{*}(s)\right) \omega(t+s) d s \\
& +\frac{1}{2} \int_{0}^{a(y)} \varphi\left(\left(y \chi_{A_{t}}\right)^{*}(s)\right) \omega(t+s) d s \\
= & \frac{1}{2} \int_{t}^{t+a(x)} \varphi\left(\left(x \chi_{A_{t}}\right)^{*}(s-t)\right) \omega(s) d s \\
& +\frac{1}{2} \int_{t}^{t+a(y)} \varphi\left(\left(y \chi_{A_{t}}\right)^{*}(s-t)\right) \omega(s) d s .
\end{aligned}
$$

\section{Results}

We start with the following

Theorem 2.1 Let $\gamma=\infty$. Then the Orlicz-Lorentz function space $\Lambda_{\varphi, \omega}$ is non-square if and only if $\int_{0}^{\infty} \omega(t) d t=\infty, \varphi \in \Delta_{2}(\mathbb{R})$ and $\int_{0}^{\gamma_{0} / 2} \varphi(\delta) \omega(t) d t<1$.

Proof Necessity. If $\int_{0}^{\infty} \omega(t) d t<\infty$ or $\varphi \notin \Delta_{2}(\mathbb{R})$, then $\Lambda_{\varphi, \omega}$ contains an order isometric copy of $l^{\infty}$ (see [26, Theorem 2.4]). Finally, suppose that $\int_{0}^{\gamma_{0} / 2} \varphi(\delta) \omega(t) d t \geq 1$. Taking

$$
x=a \chi_{\left[0, \gamma_{0} / 2\right)} \text { and } y=a \chi_{\left[\gamma_{0} / 2, \gamma_{0}\right)},
$$

where $a \leq \delta$ is such that $\int_{0}^{\gamma_{0} / 2} \varphi(a) \omega(t) d t=1$, we get $I_{\varphi, \omega}(x)=I_{\varphi, \omega}(y)=I_{\varphi, \omega}\left(\frac{x+y}{2}\right)=I_{\varphi, \omega}\left(\frac{x-y}{2}\right)=$ 1 and, consequently, $\|x\|=\|y\|=\left\|\frac{x+y}{2}\right\|=\left\|\frac{x-y}{2}\right\|=1$. Thus, $\Lambda_{\varphi, \omega}$ is not non-square.

Sufficiency. Let $x, y \in S\left(\Lambda_{\varphi, \omega}\right)$. Since $\varphi$ satisfies the condition $\Delta_{2}(\mathbb{R})$, by Lemma 1.1, it is enough to show that $\min \left(I_{\varphi, \omega}\left(\frac{x-y}{2}\right), I_{\varphi, \omega}\left(\frac{x+y}{2}\right)\right)<1$. Let us denote

$$
\begin{aligned}
& A_{1}=\{t \in(0, \infty): x(t) y(t)>0\}, \\
& A_{2}=\{t \in(0, \infty): x(t) y(t)<0\}, \\
& A_{3}=\{t \in(0, \infty): x(t) y(t)=0 \text { and } \max (|x(t)|,|y(t)|)>\delta\}, \\
& A_{4}=\{t \in(0, \infty): x(t) y(t)=0 \text { and } 0<\max (|x(t)|,|y(t)|) \leq \delta\} .
\end{aligned}
$$

By $\varphi \in \Delta_{2}(\mathbb{R})$, we have $a_{\varphi}=0$ and $b_{\varphi}=\infty$. Therefore,

$$
\varphi\left(\frac{u-v}{2}\right)<\varphi\left(\frac{\max (|u|,|v|)}{2}\right)<\frac{1}{2}\{\varphi(u)+\varphi(v)\}
$$


if $u v>0$ and

$$
\varphi\left(\frac{u+v}{2}\right)<\varphi\left(\frac{\max (|u|,|v|)}{2}\right)<\frac{1}{2}\{\varphi(u)+\varphi(v)\}
$$

whenever $u v<0$. Moreover, if $u>\delta$, then $\varphi\left(\frac{u}{2}\right)<\frac{1}{2} \varphi(u)$. Consequently,

$$
\begin{aligned}
& \varphi \circ\left(\frac{x-y}{2}\right) \supsetneqq \frac{1}{2}\{\varphi \circ(x)+\varphi \circ(y)\} \quad \text { if } m\left(A_{1}\right)>0, \\
& \varphi \circ\left(\frac{x+y}{2}\right) \supsetneqq \frac{1}{2}\{\varphi \circ(x)+\varphi \circ(y)\} \quad \text { if } m\left(A_{2} \cup A_{3}\right)>0 .
\end{aligned}
$$

Hence, by strict monotonicity of the Lorentz space $\Lambda_{\omega}$ (see Theorem 1.1), we get

$$
\begin{aligned}
& I_{\varphi, \omega}\left(\frac{x-y}{2}\right)=\left\|\varphi \circ\left(\frac{x-y}{2}\right)\right\|_{\omega}<\left\|\frac{1}{2} \varphi \circ x+\frac{1}{2} \varphi \circ y\right\|_{\omega} \leq 1 \quad \text { if } m\left(A_{1}\right)>0, \\
& I_{\varphi, \omega}\left(\frac{x+y}{2}\right)=\left\|\varphi \circ\left(\frac{x+y}{2}\right)\right\|_{\omega}<\left\|\frac{1}{2} \varphi \circ x+\frac{1}{2} \varphi \circ y\right\|_{\omega} \leq 1 \quad \text { if } m\left(A_{2} \cup A_{3}\right)>0 .
\end{aligned}
$$

Therefore, if $m\left(A_{1} \cup A_{2} \cup A_{3}\right)>0$, we have $\min \left(I_{\varphi, \omega}\left(\frac{x-y}{2}\right), I_{\varphi, \omega}\left(\frac{x+y}{2}\right)\right)<1$.

Finally, suppose that $m\left(A_{1} \cup A_{2} \cup A_{3}\right)=0$. Then $\delta>0$ and $I_{\varphi, \omega}\left(\frac{x-y}{2}\right)=I_{\varphi, \omega}\left(\frac{x+y}{2}\right)$. We will prove that

$$
\begin{aligned}
I_{\varphi, \omega}\left(\frac{x \pm y}{2}\right) & =\int_{0}^{\infty} \varphi\left(\left(\frac{x \pm y}{2}\right)^{*}(t)\right) \omega(t) d t \\
& <\frac{1}{2} \int_{0}^{\infty} \varphi\left(x^{*}(t)\right) \omega(t) d t+\frac{1}{2} \int_{0}^{\infty} \varphi\left(y^{*}(t)\right) \omega(t) d t=1 .
\end{aligned}
$$

In order to do this, we will consider two cases.

Case 1. Suppose that $\gamma_{0}>0$. Since $I_{\varphi, \omega}(x)=I_{\varphi, \omega}(y)=1$, by the condition $\int_{0}^{\gamma_{0} / 2} \varphi(\delta) \omega(t) d t<$ 1 , we have $m(\operatorname{supp} x)>\gamma_{0} / 2$ and $m(\operatorname{supp} y)>\gamma_{0} / 2$. Hence, by $m(\operatorname{supp} x \cap \operatorname{supp} y)=0$, we obtain $m(\operatorname{supp} x \cup \operatorname{supp} y)>\gamma_{0}$. By the condition $\int_{0}^{\infty} \omega(t) d t=\infty$, we have $\lim _{t \rightarrow \infty}\left(\frac{x+y}{2}\right)^{*}(t)=$ $\left(\frac{x+y}{2}\right)^{*}(\infty)=0$, whence we get $\left(\frac{x+y}{2}\right)^{*}\left(\gamma_{0}\right)>\left(\frac{x+y}{2}\right)^{*}(\infty)$. Then there exists a set $e_{\gamma_{0}}=e_{\gamma_{0}}\left(\frac{x+y}{2}\right)$ with $m\left(e_{\gamma_{0}}\right)=\gamma_{0}$ and

$$
\int_{0}^{\gamma_{0}}\left(\frac{x+y}{2}\right)^{*}(t) d t=\int_{e_{\gamma_{0}}}\left|\frac{x+y}{2}\right|(t) d t
$$

(see $\left[16\right.$, Property $7^{\circ}$, p.64]). Defining

$$
\gamma_{0}(x)=m\left(e_{\gamma_{0}} \cap \operatorname{supp} x\right) \quad \text { and } \quad \gamma_{0}(y)=m\left(e_{\gamma_{0}} \cap \operatorname{supp} y\right) \text {, }
$$

we have $\gamma_{0}(x)+\gamma_{0}(y)=\gamma_{0}$ and, by convexity of the modular $I_{\varphi, \omega}$,

$$
\begin{aligned}
\int_{0}^{\gamma_{0}} \varphi\left(\left(\frac{x+y}{2}\right)^{*}(t)\right) \omega(t) d t & =I_{\varphi, \omega}\left(\left(\frac{x+y}{2}\right) \chi_{e_{\gamma_{0}}}\right) \leq \frac{1}{2} I_{\varphi, \omega}\left(x \chi_{e_{\gamma_{0}}}\right)+\frac{1}{2} I_{\varphi, \omega}\left(y \chi_{e_{\gamma_{0}}}\right) \\
& =\frac{1}{2} \int_{0}^{\gamma_{0}(x)} \varphi\left(x^{*}(t)\right) \omega(t) d t+\frac{1}{2} \int_{0}^{\gamma_{0}(y)} \varphi\left(y^{*}(t)\right) \omega(t) d t
\end{aligned}
$$


Setting $A_{\gamma_{0}}=[0, \gamma) \backslash e_{\gamma_{0}}$, by inequality (2) from Remark 1.1, we get

$$
\begin{aligned}
& \int_{\gamma_{0}}^{\infty} \varphi\left(\left(\frac{x+y}{2}\right)^{*}(t)\right) \omega(t) d t \\
& \quad \leq \frac{1}{2} \int_{\gamma_{0}}^{\infty} \varphi\left(\left(x \chi_{A_{\gamma_{0}}}\right)^{*}\left(t-\gamma_{0}\right)\right) \omega(t) d t+\frac{1}{2} \int_{\gamma_{0}}^{\infty} \varphi\left(\left(y \chi_{A_{\gamma_{0}}}\right)^{*}\left(t-\gamma_{0}\right)\right) \omega(t) d t .
\end{aligned}
$$

Since $\varphi\left(\left(\frac{x+y}{2}\right)^{*}\left(\gamma_{0}\right)\right)>0$, we may assume without loss of generality that

$$
\int_{\gamma_{0}}^{\infty} \varphi\left(\left(x \chi_{A_{\gamma_{0}}}\right)^{*}\left(t-\gamma_{0}\right)\right) \omega(t) d t>0
$$

Denote $\omega(t)=\omega$ for $t \in\left(0, \gamma_{0}\right)$. If $\gamma_{0}(x)<\gamma_{0}$, applying the inequality $\omega(t)<\omega$ for $t>\gamma_{0}$, we get

$$
\begin{aligned}
& \frac{1}{2} \int_{0}^{\gamma_{0}(x)} \varphi\left(x^{*}(t)\right) \omega(t) d t+\frac{1}{2} \int_{\gamma_{0}}^{\infty} \varphi\left(\left(x \chi_{A_{\gamma_{0}}}\right)^{*}\left(t-\gamma_{0}\right)\right) \omega(t) d t \\
& \quad<\frac{1}{2} \int_{0}^{\gamma_{0}(x)} \varphi\left(x^{*}(t)\right) \omega(t) d t+\frac{1}{2} \int_{\gamma_{0}(x)}^{\infty} \varphi\left(\left(x \chi_{A_{\gamma_{0}}}\right)^{*}\left(t-\gamma_{0}(x)\right)\right) \omega(t) d t \\
& \quad=\frac{1}{2} \int_{0}^{\infty} \varphi\left(x^{*}(t)\right) \omega(t) d t .
\end{aligned}
$$

Suppose now that $\gamma_{0}(x)=\gamma_{0}$. Then $\gamma_{0}(y)=0$, whence supp $y \subset A_{\gamma_{0}}$ and consequently,

$$
0<\frac{1}{2} \int_{\gamma_{0}}^{\infty} \varphi\left(\left(y \chi_{A_{\gamma_{0}}}\right)^{*}\left(t-\gamma_{0}\right)\right) \omega(t) d t<\frac{1}{2} \int_{0}^{\infty} \varphi\left(y^{*}(t)\right) \omega(t) d t
$$

Applying inequalities (5), (6), (7) and (8), we obtain (4).

Case 2. Let now $\gamma_{0}=0$. Then there exists $v$ such that $\left(\frac{x+y}{2}\right)^{*}(v)>0$ and $\omega(t)>\omega(s)$ for any $t$ and $s$ satisfying $t<v<s$. Proceeding similarly as in the above Case 1, but with $v$ instead of $\gamma_{0}$, we get again inequality (4).

Theorem 2.2 If $\gamma<\infty$, then the Orlicz-Lorentz function space $\Lambda_{\varphi, \omega}$ is non-square if and only if $\frac{\gamma}{2}<\alpha \leq \gamma, \varphi \in \Delta_{2}(\infty)$ and $\int_{0}^{\gamma_{0} / 2} \varphi(\delta) \omega(t) d t<1$.

Proof Necessity. The necessity of conditions $\varphi \in \Delta_{2}(\infty)$ and $\int_{0}^{\gamma_{0} / 2} \varphi(\delta) \omega(t) d t<1$ can be shown similarly as in Theorem 2.1. Suppose that $\alpha \leq \frac{\gamma}{2}$. Since $\varphi \in \Delta_{2}(\infty)$, so $b_{\varphi}=\infty$, whence we can find $a>0$ such that $\int_{0}^{\alpha} \varphi(a) \omega(t) d t=1$. Putting

$$
\begin{aligned}
& x=a \chi_{[0,2 \alpha)}, \\
& y=a \chi_{[0, \alpha)}-a \chi_{[\alpha, 2 \alpha)},
\end{aligned}
$$

we have

$$
I_{\varphi, \omega}(x)=I_{\varphi, \omega}(y)=I_{\varphi, \omega}\left(\frac{x+y}{2}\right)=I_{\varphi, \omega}\left(\frac{x-y}{2}\right)=1,
$$

which means that $\Lambda_{\varphi, \omega}$ is not non-square. 
Sufficiency. Let $x, y \in S\left(\Lambda_{\varphi, \omega}\right)$. Analogously as in the proof of Theorem 2.1, it is enough to show that $\min \left(I_{\varphi, \omega}\left(\frac{x-y}{2}\right), I_{\varphi, \omega}\left(\frac{x+y}{2}\right)\right)<1$. We divide the proof into several parts.

Case 1. Assume that $\alpha=\gamma$. Let us define the sets $A_{i}, i=1, \ldots, 4$ as in (3) and

$$
\begin{aligned}
& A_{1}^{\prime}=\left\{t \in A_{1}: \max (|x(t)|,|y(t)|)>a_{\varphi}\right\}, \\
& A_{2}^{\prime}=\left\{t \in A_{2}: \max (|x(t)|,|y(t)|)>a_{\varphi}\right\} .
\end{aligned}
$$

If $m\left(A_{1}^{\prime}\right)>0$, then

$$
\begin{aligned}
0 & =\varphi\left(\frac{x(t)-y(t)}{2}\right)=\varphi\left(\frac{\max (|x(t)|,|y(t)|)}{2}\right)<\frac{1}{2} \varphi(\max (|x(t)|,|y(t)|)) \\
& \leq \frac{1}{2}\{\varphi(x(t))+\varphi(y(t))\}
\end{aligned}
$$

for $t \in A_{1}^{\prime}$ whenever $\max (|x(t)|,|y(t)|) / 2 \leq a_{\varphi}$ and

$$
\varphi\left(\frac{x(t)-y(t)}{2}\right)<\varphi\left(\frac{\max (|x(t)|,|y(t)|)}{2}\right) \leq \frac{1}{2}\{\varphi(x(t))+\varphi(y(t))\}
$$

for $t \in A_{1}^{\prime}$ whenever $\max (|x(t)|,|y(t)|) / 2>a_{\varphi}$. Analogously as in Theorem 2.1, by strict monotonicity of the Lorentz space $\Lambda_{\omega}$ (see Theorem 1.1), we have $I_{\varphi, \omega}\left(\frac{x-y}{2}\right)<1$. Similarly, $I_{\varphi, \omega}\left(\frac{x+y}{2}\right)<1$ provided $m\left(A_{2}^{\prime}\right)>0$. Notice that if $0=m\left(A_{1}^{\prime} \cup A_{2}^{\prime}\right)<m\left(A_{1} \cup A_{2}\right)$, then $\delta=a_{\varphi}>0$, whence $m\left(A_{3}\right)>0$ (because $\left.I_{\varphi, \omega}(x)=I_{\varphi, \omega}(y)=1\right)$. Now we will consider the case $m\left(A_{3}\right)>0$. Then

$$
\begin{aligned}
\varphi\left(\frac{x(t) \pm y(t)}{2}\right) & =\varphi\left(\frac{\max (|x(t)|,|y(t)|)}{2}\right)<\frac{1}{2} \varphi(\max (|x(t)|,|y(t)|)) \\
& =\frac{1}{2}\{\varphi(x(t))+\varphi(y(t))\}
\end{aligned}
$$

for $t \in A_{3}$, whence by strict monotonicity of the Lorentz space $\Lambda_{\omega}$, we have again $I_{\varphi, \omega}\left(\frac{x \pm y}{2}\right)<1$. Finally, suppose that $m\left(A_{1} \cup A_{2} \cup A_{3}\right)=0$. Then $0=a_{\varphi}<\delta$ and $I_{\varphi, \omega}\left(x \chi_{A_{4}}\right)=$ $I_{\varphi, \omega}\left(y \chi_{A_{4}}\right)=1$. Analogously as in the proof of Theorem 2.1, we can show

$$
I_{\varphi, \omega}\left(\frac{x \pm y}{2}\right)<\frac{1}{2} \int_{0}^{\gamma} \varphi\left(x^{*}(t)\right) \omega(t) d t+\frac{1}{2} \int_{0}^{\gamma} \varphi\left(y^{*}(t)\right) \omega(t) d t=1 .
$$

Case 2. Now suppose that $\frac{\gamma}{2}<\alpha<\gamma$ and denote

$$
A_{x, y}=\left\{t \in[0, \gamma): \max (|x(t)|,|y(t)|)>a_{\varphi}\right\} .
$$

Case 2.1. If $m\left(A_{x, y}\right) \leq \alpha$, then we define

$$
\tilde{x}=x \chi_{A_{x, y}} \circ \sigma \text { and } \tilde{y}=y \chi_{A_{x, y}} \circ \sigma \text {, }
$$

where $\sigma:\left[0, m\left(A_{x, y}\right)\right) \rightarrow A_{x, y}$ is a measure preserving transformation (see [54, Theorem 17 , p.410]). Obviously, $\varphi \circ \tilde{x}, \varphi \circ \tilde{y}, \varphi \circ \frac{\tilde{x}+\tilde{y}}{2}$ and $\varphi \circ \frac{\tilde{x}-\tilde{y}}{2}$ are equimeasurable with $\varphi \circ x \chi_{A_{x, y}}$, 
$\varphi \circ y \chi_{A_{x, y}}, \varphi \circ \frac{x+y}{2} \chi_{A_{x, y}}$ and $\varphi \circ \frac{x-y}{2} \chi_{A_{x, y},}$, respectively. Since $\Lambda_{\omega}([0, \alpha))$ is strictly monotone, repeating the proof from Case 1 , we get

$$
\begin{aligned}
\min \left(I_{\varphi, \omega}\left(\frac{x-y}{2}\right), I_{\varphi, \omega}\left(\frac{x+y}{2}\right)\right) & =\min \left(I_{\varphi, \omega}\left(\left(\frac{x-y}{2}\right) \chi_{A_{x, y}}\right), I_{\varphi, \omega}\left(\left(\frac{x+y}{2}\right) \chi_{A_{x, y}}\right)\right) \\
& =\min \left(I_{\varphi, \omega}\left(\frac{\widetilde{x}-\widetilde{y}}{2}\right), I_{\varphi, \omega}\left(\frac{\widetilde{x}+\widetilde{y}}{2}\right)\right)<1 .
\end{aligned}
$$

Case 2.2. Assume now that $m\left(A_{x, y}\right)>\alpha$, that is,

$$
\left(\frac{1}{2} \varphi \circ x+\frac{1}{2} \varphi \circ y\right)^{*}(\alpha)>0
$$

By convexity of $\varphi$ and appropriate properties of the rearrangement (see [15, Proposition 1.7, p.41]), we obtain

$$
\varphi\left(\left(\frac{x \pm y}{2}\right)^{*}(t)\right)=\left(\varphi \circ\left(\frac{x \pm y}{2}\right)\right)^{*}(t) \leq\left(\frac{1}{2} \varphi \circ x+\frac{1}{2} \varphi \circ y\right)^{*}(t)
$$

for any $t \in[0, \gamma)$. If there exists $t \in[0, \alpha)$ such that inequality (11) is sharp for the sum or for the difference, then by the right continuity of the rearrangement, we get

$$
\min \left(I_{\varphi, \omega}\left(\frac{x-y}{2}\right), I_{\varphi, \omega}\left(\frac{x+y}{2}\right)\right)<1
$$

Consequently, in the remaining part of the proof, we will assume that for any $t \in[0, \alpha)$ in formula (11), we have equality for both the sum and the difference.

Case 2.2.1. Let $\left(\frac{1}{2} \varphi \circ x+\frac{1}{2} \varphi \circ y\right)^{*}(0)>\left(\frac{1}{2} \varphi \circ x+\frac{1}{2} \varphi \circ y\right)^{*}(t)$ for all $t>\alpha$ and let us set in this case

$$
t_{0}=\sup \left\{s:\left(\frac{1}{2} \varphi \circ x+\frac{1}{2} \varphi \circ y\right)^{*}(s)>\left(\frac{1}{2} \varphi \circ x+\frac{1}{2} \varphi \circ y\right)^{*}(t) \text { for each } t>\alpha\right\} .
$$

By the right continuity of the rearrangement, we have $0<t_{0} \leq \alpha$ and

$$
\left(\frac{1}{2} \varphi \circ x+\frac{1}{2} \varphi \circ y\right)^{*}\left(t_{0}\right)=\left(\frac{1}{2} \varphi \circ x+\frac{1}{2} \varphi \circ y\right)^{*}(\alpha)>0 .
$$

Moreover, if $t_{0}=\alpha$, then $\left(\frac{1}{2} \varphi \circ x+\frac{1}{2} \varphi \circ y\right)^{*}(s)>\left(\frac{1}{2} \varphi \circ x+\frac{1}{2} \varphi \circ y\right)^{*}(\alpha)$ for any $s<\alpha$ or $\left(\frac{1}{2} \varphi \circ x+\frac{1}{2} \varphi \circ y\right)^{*}(\alpha)>\left(\frac{1}{2} \varphi \circ x+\frac{1}{2} \varphi \circ y\right)^{*}(t)$ for all $t>\alpha$. In the case when $t_{0}<\alpha$, there exists $t>\alpha$ such that $\left(\frac{1}{2} \varphi \circ x+\frac{1}{2} \varphi \circ y\right)^{*}(s)>\left(\frac{1}{2} \varphi \circ x+\frac{1}{2} \varphi \circ y\right)^{*}\left(t_{0}\right)=\left(\frac{1}{2} \varphi \circ x+\frac{1}{2} \varphi \circ y\right)^{*}(t)$ for any $s<t_{0}$. Let $e_{t_{0}}=e_{t_{0}}\left(\frac{1}{2} \varphi \circ x+\frac{1}{2} \varphi \circ y\right)$ be the set such that $m\left(e_{t_{0}}\right)=t_{0}$ and

$$
\int_{0}^{t_{0}}\left(\frac{1}{2} \varphi \circ x+\frac{1}{2} \varphi \circ y\right)^{*}(t) d t=\int_{e_{t_{0}}}\left(\frac{1}{2} \varphi \circ x+\frac{1}{2} \varphi \circ y\right)(t) d t
$$

(see $\left[16\right.$, Property $\left.7^{\circ}, \mathrm{p} .64\right]$ ). By the proof of Property $7^{\circ}$ from $[16]$, we conclude that

$$
\left(\frac{1}{2} \varphi \circ x+\frac{1}{2} \varphi \circ y\right)(s) \geq \lim _{t \rightarrow t_{0}-}\left(\frac{1}{2} \varphi \circ x+\frac{1}{2} \varphi \circ y\right)^{*}(t)
$$


for $m$-a.e. $s \in e_{t_{0}}$. Hence, by the definition of $t_{0}$, we obtain

$$
\left(\frac{1}{2} \varphi \circ x+\frac{1}{2} \varphi \circ y\right)(s)>\left(\frac{1}{2} \varphi \circ x+\frac{1}{2} \varphi \circ y\right)^{*}(t)
$$

for $m$-a.e. $s \in e_{t_{0}}$ and each $t>t_{0}$. Moreover, using again the definition of $t_{0}$, we get that for $m$-a.e. $s \in[0, \gamma) \backslash e_{t_{0}}$, there exists $t(s)>t_{0}$ such that

$$
\left(\frac{1}{2} \varphi \circ x+\frac{1}{2} \varphi \circ y\right)(s) \leq\left(\frac{1}{2} \varphi \circ x+\frac{1}{2} \varphi \circ y\right)^{*}(t(s))
$$

Since for any $t \in[0, \alpha)$ we have equality in formula (11) for both the sum and the difference, we can find sets $e_{t_{0}}(+)=e_{t_{0}}\left(\varphi \circ\left(\frac{x+y}{2}\right)\right)$ and $e_{t_{0}}(-)=e_{t_{0}}\left(\varphi \circ\left(\frac{x-y}{2}\right)\right)$ such that $m\left(e_{t_{0}}(+)\right)=$ $m\left(e_{t_{0}}(-)\right)=t_{0}$ and

$$
\int_{0}^{t_{0}}\left(\frac{1}{2} \varphi \circ x+\frac{1}{2} \varphi \circ y\right)^{*}(t) d t=\int_{e_{t_{0}}(+)} \varphi \circ\left(\frac{x+y}{2}\right)(t) d t=\int_{e_{t_{0}}(-)} \varphi \circ\left(\frac{x-y}{2}\right)(t) d t
$$

Similarly as in the case of the set $e_{t_{0}}$, for $m$-a.e. $s \in e_{t_{0}}(+)$ and for each $t>t_{0}$, we get

$$
\varphi \circ\left(\frac{x+y}{2}\right)(s)>\left(\frac{1}{2} \varphi \circ x+\frac{1}{2} \varphi \circ y\right)^{*}(t)
$$

Hence, by convexity of the function $\varphi$ and inequalities (14) and (15), we obtain $e_{t_{0}}(+) \subset e_{t_{0}}$. Since $m\left(e_{t_{0}}\right)=t_{0}=m\left(e_{t_{0}}(+)\right)$, so $e_{t_{0}}(+)=e_{t_{0}}$. Analogously, we derive the equality $e_{t_{0}}(-)=$ $e_{t_{0}}$. Note also that convexity of the function $\varphi$ and equations (13) and (16) imply the equalities

$$
\varphi \circ\left(\frac{x+y}{2}\right) \chi_{e_{0}}=\varphi \circ\left(\frac{x-y}{2}\right) \chi_{e_{t_{0}}}=\left(\frac{1}{2} \varphi \circ x+\frac{1}{2} \varphi \circ y\right) \chi_{e_{t_{0}}},
$$

whence, by inequality (10), we get $m\left(\operatorname{supp}\left(x \chi_{e_{t_{0}}}\right) \cap \operatorname{supp}\left(y \chi_{e_{t_{0}}}\right)\right)=0$ and

$$
0=a_{\varphi}<\left(\frac{1}{2} \varphi \circ x+\frac{1}{2} \varphi \circ y\right)^{*}(0) \leq \delta .
$$

Denoting $t_{0}(x)=m\left(e_{t_{0}} \cap \operatorname{supp} x\right)$ and $t_{0}(y)=m\left(e_{t_{0}} \cap \operatorname{supp} y\right)$, we have

$$
t_{0}(x)+t_{0}(y)=t_{0}
$$

Case 2.2.1.1. Suppose $t_{0}=\alpha$. By convexity of the modular $I_{\varphi, \omega}$, we get

$$
\begin{aligned}
\int_{0}^{t_{0}} \varphi\left(\left(\frac{x+y}{2}\right)^{*}(t)\right) \omega(t) d t & =I_{\varphi, \omega}\left(\left(\frac{x+y}{2}\right) \chi_{e_{t_{0}}}\right) \leq \frac{1}{2} I_{\varphi, \omega}\left(x \chi_{e_{t_{0}}}\right)+\frac{1}{2} I_{\varphi, \omega}\left(y \chi_{e_{t_{0}}}\right) \\
& =\frac{1}{2} \int_{0}^{t_{0}(x)} \varphi\left(x^{*}(t)\right) \omega(t) d t+\frac{1}{2} \int_{0}^{t_{0}(y)} \varphi\left(y^{*}(t)\right) \omega(t) d t .
\end{aligned}
$$

If $t_{0}(y)=0\left(t_{0}(x)=0\right)$, then $I_{\varphi, \omega}\left(\frac{x+y}{2}\right) \leq \frac{1}{2} I_{\varphi, \omega}(x)=\frac{1}{2}\left(I_{\varphi, \omega}\left(\frac{x+y}{2}\right) \leq \frac{1}{2} I_{\varphi, \omega}(y)=\frac{1}{2}\right)$. So, $0<t_{0}(x)<$ $t_{0}$ and $0<t_{0}(y)<t_{0}$. Furthermore, by equation (10), we may assume without loss of gener- 
ality that $\beta(x):=m\left(\left(A_{x, y} \backslash e_{t_{0}}\right) \cap \operatorname{supp} x\right)>0$. Thus

$$
\begin{aligned}
\int_{0}^{t_{0}(x)} \varphi\left(x^{*}(t)\right) \omega(t) d t & <\int_{0}^{t_{0}(x)} \varphi\left(x^{*}(t)\right) \omega(t) d t \\
& +\int_{t_{0}(x)}^{t_{0}(x)+\beta(x)} \varphi\left(\left(x \chi_{A_{x, y} \backslash e_{0}}\right)^{*}\left(t-t_{0}(x)\right)\right) \omega(t) d t \\
= & \int_{0}^{\alpha} \varphi\left(x^{*}(t)\right) \omega(t) d t=1,
\end{aligned}
$$

whence we get $I_{\varphi, \omega}\left(\frac{x+y}{2}\right)<1$.

Case 2.2.1.2. Let now $t_{0}<\alpha$. Then, by the definition of $t_{0}$, there exists $t>\alpha$ satisfying

$$
\left(\frac{1}{2} \varphi \circ x+\frac{1}{2} \varphi \circ y\right)^{*}(t)=\left(\frac{1}{2} \varphi \circ x+\frac{1}{2} \varphi \circ y\right)^{*}\left(t_{0}\right) .
$$

Define

$$
\begin{aligned}
& t_{1}=\sup \left\{t>\alpha:\left(\frac{1}{2} \varphi \circ x+\frac{1}{2} \varphi \circ y\right)^{*}(t)=\left(\frac{1}{2} \varphi \circ x+\frac{1}{2} \varphi \circ y\right)^{*}\left(t_{0}\right)\right\} \\
& A_{t_{0}}=\left\{t \in[0, \gamma):\left(\frac{1}{2} \varphi \circ x+\frac{1}{2} \varphi \circ y\right)(t)=\left(\frac{1}{2} \varphi \circ x+\frac{1}{2} \varphi \circ y\right)^{*}\left(t_{0}\right)\right\}
\end{aligned}
$$

and

$$
A_{t_{0}, x, y}=\left\{t \in A_{t_{0}}: \min (|x(t)|,|y(t)|)=0\right\} .
$$

Since for any $t \in[0, \alpha)$ we have equality in formula (11) for both the sum and the difference, we can find a set $e_{\alpha}=e_{\alpha}\left(\frac{1}{2} \varphi \circ x+\frac{1}{2} \varphi \circ y\right)$ such that $m\left(e_{\alpha}\right)=\alpha$ and

$$
\int_{0}^{\alpha}\left(\frac{1}{2} \varphi \circ x+\frac{1}{2} \varphi \circ y\right)^{*}(t) d t=\int_{e_{\alpha}}\left(\frac{1}{2} \varphi \circ x+\frac{1}{2} \varphi \circ y\right)(t) d t=\int_{e_{\alpha}} \varphi \circ\left(\frac{x+y}{2}\right)(t) d t .
$$

If $m\left(A_{t_{0}, x, y}\right) \geq \alpha-t_{0}$, then we can assume without loss of generality that $e_{t_{0}} \subset e_{\alpha} \subset e_{t_{0}} \cup$ $A_{t_{0}, x, y}$, whence we get the equality $m\left(\operatorname{supp} x \chi_{e_{\alpha}} \cap \operatorname{supp} y \chi_{e_{\alpha}}\right)=0$. Proceeding analogously as in Case 2.2.1.1, we obtain $I_{\varphi, \omega}\left(\frac{x+y}{2}\right)<1$.

Let now $m\left(A_{t_{0}, x, y}\right)<\alpha-t_{0}$. Then we will suppose that $e_{t_{0}} \cup A_{t_{0}, x, y} \subset e_{\alpha} \subset e_{t_{0}} \cup A_{t_{0}}$ and consequently

$$
m\left(\left(A_{t_{0}} \backslash e_{\alpha}\right) \cap \operatorname{supp} x\right)=m\left(\left(A_{t_{0}} \backslash e_{\alpha}\right) \cap \operatorname{supp} y\right)=m\left(A_{t_{0}} \backslash e_{\alpha}\right)=t_{1}-\alpha=: d>0 .
$$

Putting $\alpha(x)=m\left(e_{\alpha} \cap \operatorname{supp} x\right), \alpha(y)=m\left(e_{\alpha} \cap \operatorname{supp} y\right)$ and applying again convexity of the modular $I_{\varphi, \omega}$, we obtain

$$
\begin{aligned}
\int_{0}^{\alpha} \varphi\left(\left(\frac{x+y}{2}\right)^{*}(t)\right) \omega(t) d t & =I_{\varphi, \omega}\left(\left(\frac{x+y}{2}\right) \chi_{e_{\alpha}}\right) \leq \frac{1}{2} I_{\varphi, \omega}\left(x \chi_{e_{\alpha}}\right)+\frac{1}{2} I_{\varphi, \omega}\left(y \chi_{e_{\alpha}}\right) \\
= & \frac{1}{2} \int_{0}^{\alpha(x)} \varphi\left(\left(x \chi_{e_{\alpha}}\right)^{*}(t)\right) \omega(t) d t \\
& +\frac{1}{2} \int_{0}^{\alpha(y)} \varphi\left(\left(y \chi_{e_{\alpha}}\right)^{*}(t)\right) \omega(t) d t
\end{aligned}
$$


Simultaneously, by equality (18), we may assume without loss of generality that $\alpha(x)=$ $t_{0}(x)+m\left(\left(e_{\alpha} \backslash e_{t_{0}}\right) \cap \operatorname{supp} x\right)<\alpha$, whence

$$
\begin{aligned}
\int_{0}^{\alpha(x)} \varphi\left(\left(x \chi_{e_{\alpha}}\right)^{*}(t)\right) \omega(t) d t< & \int_{0}^{\alpha(x)} \varphi\left(\left(x \chi_{e_{\alpha}}\right)^{*}(t)\right) \omega(t) d t \\
& +\int_{\alpha(x)}^{\alpha(x)+d} \varphi\left(\left(x \chi_{A_{t_{0}} \backslash e_{\alpha}}\right)^{*}(t-\alpha(x))\right) \omega(t) d t \\
\leq & \int_{0}^{\alpha} \varphi\left(x^{*}(t)\right) \omega(t) d t=1 .
\end{aligned}
$$

So, we get $I_{\varphi, \omega}\left(\frac{x+y}{2}\right)<1$.

Case 2.2.2. Finally, assume that $\left(\frac{1}{2} \varphi \circ x+\frac{1}{2} \varphi \circ y\right)^{*}(0)=\left(\frac{1}{2} \varphi \circ x+\frac{1}{2} \varphi \circ y\right)^{*}(\alpha)=\left(\frac{1}{2} \varphi \circ x+\right.$ $\left.\frac{1}{2} \varphi \circ y\right)^{*}(t)>0$ for some $t>\alpha$ and define

$$
\begin{aligned}
& A=\left\{t \in[0, \gamma): \frac{1}{2} \varphi(x(t))+\frac{1}{2} \varphi(y(t))=\left(\frac{1}{2} \varphi \circ x+\frac{1}{2} \varphi \circ y\right)^{*}(0)\right\}, \\
& A_{+}=\left\{t \in[0, \gamma): \varphi \circ\left(\frac{x+y}{2}\right)(t)=\left(\frac{1}{2} \varphi \circ x+\frac{1}{2} \varphi \circ y\right)^{*}(0)\right\}, \\
& A_{-}=\left\{t \in[0, \gamma): \varphi \circ\left(\frac{x-y}{2}\right)(t)=\left(\frac{1}{2} \varphi \circ x+\frac{1}{2} \varphi \circ y\right)^{*}(0)\right\} .
\end{aligned}
$$

Applying convexity of the Orlicz function and the equality in formula (11), we get the conditions $m(A)>\alpha, A_{+} \subset A, A_{-} \subset A$ and $\min \left(m\left(A_{+}\right), m\left(A_{-}\right)\right) \geq \alpha$. Since $\alpha>\frac{\gamma}{2}$, the set $A_{x, y}=A_{+} \cap A_{-}=\{t \in A: \min (|x(t)|,|y(t)|)=0\}$ has positive measure. If $m\left(A_{x, y}\right) \geq \alpha$, we can assume that $e_{\alpha} \subset A_{x, y}$ (where $e_{\alpha}$ is defined analogously as in (19)); in the opposite case, we can assume that $A_{x, y} \subset e_{\alpha} \subset A$. Proceeding analogously as in Case 2.2.1, we obtain $I_{\varphi, \omega}\left(\frac{x+y}{2}\right)<1$.

Theorem 2.3 In the case when $\gamma=\infty$, the Orlicz-Lorentz function space $\Lambda_{\varphi, \omega}$ is uniformly non-square if and only if $\varphi \in \Delta_{2}(\mathbb{R}), \psi \in \Delta_{2}(\mathbb{R})$ and $\omega$ is regular.

Proof Necessity. The necessity of the condition $\varphi \in \Delta_{2}(\mathbb{R})$ follows from Theorem 2.1. If $\psi \notin \Delta_{2}(\mathbb{R})$, then $\Lambda_{\varphi, \omega}$ contains an order isomorphic copy of $l^{1}$ (see [38, Theorem 7.18] or [29, Theorem 2]), whence it is not reflexive. Finally, suppose that $\omega$ is not regular. Then we can find a sequence $\left(t_{n}\right)$ of positive numbers such that

$$
\int_{0}^{2 t_{n}} \omega(t) d t \leq\left(1+\frac{1}{n}\right) \int_{0}^{t_{n}} \omega(t) d t
$$

for any $n \in \mathbb{N}$. Since $b_{\varphi}=\infty$, for every $n \in \mathbb{N}$, there exists $a_{n}$ satisfying

$$
\varphi\left(a_{n}\right) \int_{0}^{2 t_{n}} \omega(t) d t=1
$$

Define

$$
\begin{aligned}
& x_{n}=a_{n} \chi_{\left[0,2 t_{n}\right)}, \\
& y_{n}=a_{n} \chi_{\left[0, t_{n}\right)}-a_{n} \chi_{\left[t_{n}, 2 t_{n}\right)} .
\end{aligned}
$$


Then $I_{\varphi, \omega}\left(x_{n}\right)=I_{\varphi, \omega}\left(y_{n}\right)=1$ and

$$
I_{\varphi, \omega}\left(\frac{x_{n}+y_{n}}{2}\right)=I_{\varphi, \omega}\left(\frac{x_{n}-y_{n}}{2}\right)=\int_{0}^{t_{n}} \varphi\left(a_{n}\right) \omega(t) d t \geq \frac{n}{n+1} \int_{0}^{2 t_{n}} \varphi\left(a_{n}\right) \omega(t) d t \rightarrow 1,
$$

whence we have $\min \left(\left\|\frac{x_{n}-y_{n}}{2}\right\|,\left\|\frac{x_{n}+y_{n}}{2}\right\|\right) \rightarrow 1$.

Sufficiency. Let $x, y \in S\left(\Lambda_{\varphi, \omega}\right)$. By $\psi \in \Delta_{2}(\mathbb{R})$ we conclude that there is $\eta \in(0,1)$ such that $\varphi\left(\frac{u}{2}\right) \leq \frac{1-\eta}{2} \varphi(u)$ for all $u>0$ (see [55]). Let us set

$$
\begin{aligned}
& A_{1}=\{t \in(0, \infty): x(t) y(t)>0\}, \\
& A_{2}=\{t \in(0, \infty): x(t) y(t)<0\}, \\
& A_{3}=\{t \in(0, \infty):|x(t)|>0 \text { and } y(t)=0\} .
\end{aligned}
$$

Since $I_{\varphi, \omega}(x)=1$, we have $\max \left(I_{\varphi, \omega}\left(x \chi_{A_{1} \cup A_{3}}\right), I_{\varphi, \omega}\left(x \chi_{A_{2}}\right)\right) \geq 1 / 2$. Suppose that $I_{\varphi, \omega}\left(x \chi_{A_{1} \cup A_{3}}\right) \geq 1 / 2$. Since the inequality

$$
\begin{aligned}
\varphi\left(\frac{x(t)-y(t)}{2}\right) & \leq \varphi\left(\frac{\max (|x(t)|,|y(t)|)}{2}\right) \leq \frac{1-\eta}{2} \varphi(\max (|x(t)|,|y(t)|)) \\
& \leq \frac{1}{2} \varphi(x(t))+\frac{1}{2} \varphi(y(t))-\frac{\eta}{2} \varphi(x(t))
\end{aligned}
$$

holds for $m$-a.e. $t \in A_{1} \cup A_{3}$, we get

$$
\varphi \circ\left(\frac{x-y}{2}\right) \leq \frac{1}{2} \varphi \circ x+\frac{1}{2} \varphi \circ y-\frac{\eta}{2} \varphi \circ x \chi_{A_{1} \cup A_{3}}
$$

Hence, by uniform monotonicity of the Lorentz space $\Lambda_{\omega}$ (see Theorem 1.2), we obtain

$$
I_{\varphi, \omega}\left(\frac{x-y}{2}\right)=\left\|\varphi \circ\left(\frac{x-y}{2}\right)\right\|_{\omega} \leq\left\|\frac{1}{2} \varphi \circ x+\frac{1}{2} \varphi \circ y-\frac{\eta}{2} \varphi \circ x \chi_{A_{1} \cup A_{3}}\right\|_{\varphi} \leq 1-\delta\left(\frac{\eta}{4}\right),
$$

where $\delta\left(\frac{\eta}{4}\right)$ is the constant from the definition of uniform monotonicity of the Lorentz space $\Lambda_{\omega}$ corresponding to $\frac{\eta}{4}$. Analogously, we get $I_{\varphi, \omega}\left(\frac{x+y}{2}\right) \leq 1-\delta\left(\frac{\eta}{4}\right)$ in the case when $I_{\varphi, \omega}\left(x \chi_{A_{2}}\right) \geq 1 / 2$. Finally, by Lemma 1.1 , we obtain

$$
\min \left(\left\|\frac{x-y}{2}\right\|,\left\|\frac{x+y}{2}\right\|\right) \leq 1-r
$$

where $r=r\left(\delta\left(\frac{\eta}{4}\right)\right)$ depends only on $\delta\left(\frac{\eta}{4}\right)$.

Theorem 2.4 If $\alpha=\gamma<\infty$, then the Orlicz-Lorentz function space $\Lambda_{\varphi, \omega}$ is uniformly nonsquare if and only if $\varphi \in \Delta_{2}(\infty), \psi \in \Delta_{2}(\infty), \omega$ is regular and $\int_{0}^{\gamma_{0} / 2} \varphi(\delta) \omega(t) d t<1$.

Proof Necessity. The necessity of the conditions $\varphi \in \Delta_{2}(\infty)$ and $\int_{0}^{\gamma_{0} / 2} \varphi(\delta) \omega(t) d t<1$ has been shown in Theorem 2.2, whereas the necessity of the conditions $\psi \in \Delta_{2}(\infty)$ and regularity of $\omega$ can be shown analogously as in Theorem 2.3 .

Sufficiency. Let $x, y \in S\left(\Lambda_{\varphi, \omega}\right)$. If we show the inequality

$$
\min \left(I_{\varphi, \omega}\left(\frac{x-y}{2}\right), I_{\varphi, \omega}\left(\frac{x+y}{2}\right)\right) \leq 1-q
$$


for some $q>0$ independent of $x$ and $y$, then Lemma 1.1 will give the inequality

$$
\min \left(\left\|\frac{x-y}{2}\right\|,\left\|\frac{x+y}{2}\right\|\right) \leq 1-r
$$

with some $r>0$ depending only on $q$, and the proof will be finished. In order to show (20), we consider three cases.

Case 1. First assume that $\int_{0}^{\gamma} \varphi(\delta) \omega(t) d t<1$ (in particular, this holds if $\delta=0$ or $0<a_{\varphi}=$ $\delta)$. Then we can find $u_{\delta}>\delta$ such that $\int_{0}^{\gamma} \varphi\left(u_{\delta}\right) \omega(t) d t=: a_{\delta}<1$. Since for any $u>\delta$ there holds

$$
\varphi\left(\frac{u}{2}\right)<\frac{1}{2} \varphi(u)
$$

by $\psi \in \Delta_{2}(\infty)$, there exists $\eta=\eta\left(u_{\delta}\right) \in(0,1)$ such that

$$
\varphi\left(\frac{u}{2}\right) \leq \frac{1-\eta}{2} \varphi(u)
$$

for all $u \geq u_{\delta}$ (see [55]). Define

$$
\begin{aligned}
& A=\left\{t \in[0, \gamma):|x(t)| \geq u_{\delta}\right\}, \\
& A_{1}=\{t \in A: x(t) y(t) \geq 0\}, \\
& A_{2}=\{t \in A: x(t) y(t)<0\} .
\end{aligned}
$$

We have $I_{\varphi, \omega}\left(x \chi_{[0, \gamma) \backslash A}\right)<a_{\delta}$, whence $I_{\varphi, \omega}\left(x \chi_{A}\right)>1-a_{\delta}$ and consequently

$$
\max \left(I_{\varphi, \omega}\left(x \chi_{A_{1}}\right), I_{\varphi, \omega}\left(x \chi_{A_{2}}\right)\right)>\frac{1-a_{\delta}}{2}
$$

If $I_{\varphi, \omega}\left(x \chi_{A_{1}}\right)>\left(1-a_{\delta}\right) / 2$, analogously as in the proof of Theorem 2.3 , we get

$$
\varphi \circ \frac{x-y}{2} \leq \frac{1}{2} \varphi \circ x+\frac{1}{2} \varphi \circ y-\frac{\eta}{2} \varphi \circ x \chi_{A_{1}} .
$$

Hence, by uniform monotonicity of the Lorentz space $\Lambda_{\omega}$ (see Theorem 1.2), we obtain

$$
\begin{aligned}
I_{\varphi, \omega}\left(\frac{x-y}{2}\right) & =\left\|\varphi \circ\left(\frac{x-y}{2}\right)\right\|_{\omega} \leq\left\|\frac{1}{2} \varphi \circ x+\frac{1}{2} \varphi \circ y-\frac{\eta}{2} \varphi \circ x \chi_{A_{1}}\right\|_{\omega} \\
& \leq 1-\delta\left(\frac{\eta\left(1-a_{\delta}\right)}{4}\right),
\end{aligned}
$$

where $\delta\left(\eta\left(1-a_{\delta}\right) / 4\right)$ is the constant from the definition of uniform monotonicity of the Lorentz space $\Lambda_{\omega}$ corresponding to $\eta\left(1-a_{\delta}\right) / 4$. If $I_{\varphi, \omega}\left(x \chi_{A_{2}}\right)>\left(1-a_{\delta}\right) / 2$, then we get similarly that $I_{\varphi, \omega}\left(\frac{x+y}{2}\right) \leq 1-\delta\left(\eta\left(1-a_{\delta}\right) / 4\right)$. Therefore, if $\int_{0}^{\gamma} \varphi(\delta) \omega(t) d t<1$, we obtain inequality (20) with $q=\delta\left(\eta\left(1-a_{\delta}\right) / 4\right)$. 
Case 2. Now assume that $\int_{0}^{\gamma} \varphi(\delta) \omega(t) d t \geq 1$ and $\gamma_{0}>0$. Then for

$$
c:=\frac{1-\int_{0}^{\gamma_{0} / 2} \varphi(\delta) \omega(t) d t}{8}
$$

by the condition $\int_{0}^{\gamma_{0} / 2} \varphi(\delta) \omega(t) d t<1$, we have $0<c<\frac{1}{8}$. Moreover, we can find a constant $v_{\delta}>\delta$ such that

$$
\int_{0}^{\gamma_{0} / 2} \varphi\left(v_{\delta}\right) \omega(t) d t=1-4 c
$$

Applying again the condition $\psi \in \Delta_{2}(\infty)$, we get that there exists $\eta=\eta\left(v_{\delta}\right) \in(0,1)$ such that inequality (21) holds for any $u \geq v_{\delta}$. Denote

$$
\begin{aligned}
& A_{x, v_{\delta}}=\left\{t \in[0, \gamma):|x(t)| \geq v_{\delta}\right\}, \\
& A_{y, v_{\delta}}=\left\{t \in[0, \gamma):|y(t)| \geq v_{\delta}\right\} .
\end{aligned}
$$

Now we divide the proof of this case into several parts.

Case 2.1. If $\max \left(I_{\varphi, \omega}\left(x \chi_{A_{x, v_{\delta}}}\right), I_{\varphi, \omega}\left(y \chi_{A_{y, v_{\delta}}}\right)\right) \geq c$, then proceeding analogously as in the Case 1 , we get

$$
\min \left(I_{\varphi, \omega}\left(\frac{x-y}{2}\right), I_{\varphi, \omega}\left(\frac{x+y}{2}\right)\right) \leq 1-\delta\left(\frac{\eta c}{4}\right)
$$

where $\delta\left(\frac{\eta c}{4}\right)$ is the constant from the definition of uniform monotonicity of the Lorentz space $\Lambda_{\omega}$ corresponding to $\frac{\eta c}{4}$.

Case 2.2. Now assume that $\max \left(I_{\varphi, \omega}\left(x \chi_{A_{x, v_{\delta}}}\right), I_{\varphi, \omega}\left(y \chi_{A_{y, v_{\delta}}}\right)\right)<c$ and define $t_{0}>0$ and $u_{0}>$ 0 by the formulas

$$
\int_{0}^{t_{0}} \varphi\left(v_{\delta}\right) \omega(t) d t=1-2 c \text { and } \int_{0}^{\gamma} \varphi\left(u_{0}\right) \omega(t) d t=c
$$

By the definition of $v_{\delta}$ and the inequality $\int_{0}^{\gamma} \varphi(\delta) \omega(t) d t \geq 1$, we have $t_{0}>\frac{\gamma_{0}}{2}$ and $u_{0}<\delta$, respectively.

Now we will show that

$$
m\left(A_{x, u_{0}}\right) \geq t_{0} \quad \text { and } \quad m\left(A_{y, u_{0}}\right) \geq t_{0},
$$

where

$$
\begin{aligned}
& A_{x, u_{0}}=\left\{t \in[0, \gamma):|x(t)| \geq u_{0}\right\}, \\
& A_{y, u_{0}}=\left\{t \in[0, \gamma):|y(t)| \geq u_{0}\right\} .
\end{aligned}
$$

Indeed, by the equalities $I_{\varphi, \omega}(x)=I_{\varphi, \omega}(y)=1$ and the definition of $u_{0}$, we have $I_{\varphi, \omega}\left(x \chi_{A_{x, u_{0}}}\right) \geq$ $1-c$ and $I_{\varphi, \omega}\left(y \chi_{A_{y, u_{0}}}\right) \geq 1-c$, whence by $\max \left(I_{\varphi, \omega}\left(x \chi_{A_{x, v_{\delta}}}\right), I_{\varphi, \omega}\left(y \chi_{A_{y, v_{\delta}}}\right)\right)<c$ and the definition of $t_{0}$, we get (25). 
Let

$$
t_{1}=\frac{\min \left(t_{0}-\frac{\gamma_{0}}{2}, \frac{\gamma_{0}}{2}\right)}{4}
$$

and

$$
\begin{aligned}
& A_{x, y, u_{0}}^{+}=\left\{t \in[0, \gamma): \min (|x(t)|,|y(t)|) \geq \frac{u_{0}}{4} \text { and } x(t) y(t)>0\right\}, \\
& A_{x, y, u_{0}}^{-}=\left\{t \in[0, \gamma): \min (|x(t)|,|y(t)|) \geq \frac{u_{0}}{4} \text { and } x(t) y(t)<0\right\} .
\end{aligned}
$$

Case 2.2.1. First assume that $m\left(A_{x, y, u_{0}}^{+}\right) \geq t_{1}$ and define

$$
z_{1}=\left(\frac{1}{2} \varphi \circ x+\frac{1}{2} \varphi \circ y\right)-\varphi \circ\left(\frac{x-y}{2}\right) .
$$

Denoting by $p(u)$ the right derivative of $\varphi$ at a point $u$, we have $p(u)=: p>0$ for $u \in[0, \delta)$. Note that for $m$-a.e. $t \in A_{x, y, u_{0}}^{+}$, we have

$$
\begin{aligned}
\left(\frac{1}{2} \varphi(x(t))+\frac{1}{2} \varphi(y(t))\right)-\varphi\left(\frac{x(t)-y(t)}{2}\right) & \geq \varphi\left(\frac{x(t)+y(t)}{2}\right)-\varphi\left(\frac{x(t)-y(t)}{2}\right) \\
& \geq \int_{\varphi\left(\frac{x(t)-y(t)}{2}\right)}^{\varphi\left(\frac{x(t)+y(t)}{2}\right)} p(u) d u \geq \int_{0}^{u_{0} / 2} p d u=\frac{p u_{0}}{2} .
\end{aligned}
$$

Hence, by $m\left(A_{x, y, u_{0}}^{+}\right) \geq t_{1}$ and $t_{1}<\gamma_{0}$, we get

$$
\left\|z_{1}\right\|_{\omega} \geq \int_{0}^{t_{1}} \frac{p u_{0}}{2} \omega(t) d t=\frac{p u_{0} \omega_{0} t_{1}}{2}
$$

where $\omega_{0}=\omega(t)$ for any $t \in\left(0, \gamma_{0}\right)$. Analogously, if $m\left(A_{x, y, u_{0}}^{-}\right) \geq t_{1}$, for

$$
z_{2}=\left(\frac{1}{2} \varphi \circ x+\frac{1}{2} \varphi \circ y\right)-\varphi \circ\left(\frac{x+y}{2}\right)
$$

we obtain

$$
\left\|z_{2}\right\|_{\omega} \geq \int_{0}^{t_{1}} \frac{p u_{0}}{2} \omega(t) d t=\frac{p u_{0} \omega_{0} t_{1}}{2} .
$$

Therefore, if $\max \left(m\left(A_{x, y, u_{0}}^{+}\right), m\left(A_{x, y, u_{0}}^{-}\right)\right) \geq t_{1}$, by uniform monotonicity of the Lorentz space $\Lambda_{\omega}$, we have

$$
\min \left(I_{\varphi, \omega}\left(\frac{x-y}{2}\right), I_{\varphi, \omega}\left(\frac{x+y}{2}\right)\right) \leq 1-\delta\left(\frac{p u_{0} \omega_{0} t_{1}}{2}\right)
$$

where $\delta\left(\frac{p u_{0} \omega_{0} t_{1}}{2}\right)$ is the constant from the definition of uniform monotonicity of the Lorentz space $\Lambda_{\omega}$ corresponding to $\frac{p u_{0} \omega_{0} t_{1}}{2}$. 
Case 2.2.2. Finally, suppose that $\max \left(m\left(A_{x, y, u_{0}}^{+}\right), m\left(A_{x, y, u_{0}}^{-}\right)\right)<t_{1}$. Then for

$$
\begin{aligned}
& B_{x, u_{0}}=A_{x, u_{0}} \backslash\left(A_{x, y, u_{0}}^{+} \cup A_{x, y, u_{0}}^{-}\right), \\
& B_{y, u_{0}}=A_{y, u_{0}} \backslash\left(A_{x, y, u_{0}}^{+} \cup A_{x, y, u_{0}}^{-}\right),
\end{aligned}
$$

we have

$$
B_{x, u_{0}} \cap B_{y, u_{0}}=\emptyset
$$

and by (25) and definition of $t_{1}$

$$
\min \left(m\left(B_{x, u_{0}}\right), m\left(B_{y, u_{0}}\right)\right) \geq t_{0}-2 t_{1} \geq t_{0}-\frac{1}{2}\left(t_{0}-\frac{\gamma_{0}}{2}\right)=\frac{t_{0}}{2}+\frac{\gamma_{0}}{4}>\frac{\gamma_{0}}{2},
$$

whence we get

$$
m\left(B_{x, u_{0}} \cup B_{y, u_{0}}\right) \geq t_{0}+\frac{\gamma_{0}}{2}>\gamma_{0} .
$$

Define

$$
a=\min \left(\frac{\left(t_{0}+\frac{\gamma_{0}}{2}\right)-\gamma_{0}}{8}, \frac{\gamma_{0}}{4}\right) \text { and } t_{2}=\gamma_{0}+a
$$

Let $e_{\gamma_{0}}=e_{\gamma_{0}}\left(\frac{x+y}{2}\right)$ and $e_{t_{2}}=e_{t_{2}}\left(\frac{x+y}{2}\right)$ be such that $m\left(e_{\gamma_{0}}\right)=\gamma_{0}, m\left(e_{t_{2}}\right)=t_{2}$,

$$
\int_{0}^{\gamma_{0}}\left(\frac{x+y}{2}\right)^{*}(t) d t=\int_{e_{\gamma_{0}}}\left|\frac{x+y}{2}\right|(t) d t
$$

and

$$
\int_{0}^{t_{2}}\left(\frac{x+y}{2}\right)^{*}(t) d t=\int_{e_{t_{2}}}\left|\frac{x+y}{2}\right|(t) d t
$$

(see $\left[16\right.$, Property $\left.7^{\circ}, \mathrm{p} .64\right]$ ). Moreover, by the proof of Property $7^{\circ}$, we can assume that $e_{\gamma_{0}} \subset e_{t_{2}}$. Denoting $A_{\gamma_{0}}=e_{t_{2}} \backslash e_{\gamma_{0}}$ and $A_{t_{2}}=[0, \gamma) \backslash e_{t_{2}}$, by Remark 1.1, we have

$$
\begin{aligned}
I_{\varphi, \omega} & \left(\frac{x+y}{2}\right) \\
= & \int_{0}^{\gamma_{0}} \varphi\left(\left(\frac{x+y}{2}\right)^{*}(t)\right) \omega(t) d t+\int_{\gamma_{0}}^{t_{2}} \varphi\left(\left(\frac{x+y}{2}\right)^{*}(t)\right) \omega(t) d t \\
& +\int_{t_{2}}^{\gamma} \varphi\left(\left(\frac{x+y}{2}\right)^{*}(t)\right) \omega(t) d t \\
= & \int_{0}^{\gamma_{0}} \varphi\left(\left(\left(\frac{x+y}{2}\right) \chi_{e_{\gamma_{0}}}\right)^{*}(t)\right) \omega(t) d t+\int_{\gamma_{0}}^{t_{2}} \varphi\left(\left(\left(\frac{x+y}{2}\right) \chi_{A_{\gamma_{0}}}\right)^{*}\left(t-\gamma_{0}\right)\right) \omega(t) d t \\
& +\int_{t_{2}}^{\gamma} \varphi\left(\left(\left(\frac{x+y}{2}\right) \chi_{A_{t_{2}}}\right)^{*}\left(t-t_{2}\right)\right)^{*} \omega(t) d t \\
\leq & \frac{1}{2} \int_{0}^{\gamma_{0}} \varphi\left(\left(x \chi_{e_{\gamma_{0}}}\right)^{*}(t)\right) \omega(t) d t+\frac{1}{2} \int_{0}^{\gamma_{0}} \varphi\left(\left(y \chi_{e_{\gamma_{0}}}\right)^{*}(t)\right) \omega(t) d t
\end{aligned}
$$




$$
\begin{aligned}
& +\frac{1}{2} \int_{\gamma_{0}}^{t_{2}} \varphi\left(\left(x \chi_{A_{\gamma_{0}}}\right)^{*}\left(t-\gamma_{0}\right)\right) \omega(t) d t+\frac{1}{2} \int_{\gamma_{0}}^{t_{2}} \varphi\left(\left(y \chi_{A_{\gamma_{0}}}\right)^{*}\left(t-\gamma_{0}\right)\right) \omega(t) d t \\
& +\frac{1}{2} \int_{t_{2}}^{\gamma} \varphi\left(\left(x \chi_{A_{t_{2}}}\right)^{*}\left(t-t_{2}\right)\right) \omega(t) d t+\frac{1}{2} \int_{t_{2}}^{\gamma} \varphi\left(\left(y \chi_{A_{t_{2}}}\right)^{*}\left(t-t_{2}\right)\right) \omega(t) d t .
\end{aligned}
$$

By formulas (33) and (35), we have

$$
m\left(\left(B_{x, u_{0}} \cup B_{y, u_{0}}\right) \cap A_{t_{2}}\right)=m\left(\left(B_{x, u_{0}} \cup B_{y, u_{0}}\right) \backslash e_{t_{2}}\right) \geq t_{0}+\frac{\gamma_{0}}{2}-t_{2}=t_{0}-\frac{\gamma_{0}}{2}-a \geq 7 a
$$

and, in consequence, we can assume without loss of generality that $\left(x \chi_{A_{t_{2}}}\right)^{*}(a)>u_{0}$. If $\left(x \chi_{e_{\gamma_{0}}}\right)^{*}\left(\gamma_{0}-a\right) \leq \frac{u_{0}}{4}$, then

$$
\begin{aligned}
& \int_{\gamma_{0}-a}^{\gamma_{0}}\left[\varphi\left(\left(x \chi_{A_{t_{2}}}\right)^{*}\left(t-\gamma_{0}+a\right)\right)-\varphi\left(\left(x \chi_{e_{\gamma_{0}}}\right)^{*}(t)\right)\right] \omega(t) d t \\
& \quad-\int_{t_{2}}^{t_{2}+a}\left[\varphi\left(\left(x \chi_{A_{t_{2}}}\right)^{*}\left(t-t_{2}\right)\right)-\varphi\left(\left(x \chi_{e_{\gamma_{0}}}\right)^{*}\left(t-\left(t_{2}-\gamma_{0}+a\right)\right)\right)\right] \omega(t) d t \\
& \quad \geq\left(\omega_{0}-\omega\left(t_{2}\right)\right) \int_{\gamma_{0}-a}^{\gamma_{0}}\left(\varphi\left(\left(x \chi_{A_{t_{2}}}\right)^{*}\left(t-\gamma_{0}+a\right)\right)-\varphi\left(\left(x \chi_{e_{\gamma_{0}}}\right)^{*}(t)\right)\right) d t \\
& \geq a\left(\varphi\left(u_{0}\right)-\varphi\left(\frac{u_{0}}{4}\right)\right)\left(\omega_{0}-\omega\left(t_{2}\right)\right) \geq \frac{3 a p u_{0}\left(\omega_{0}-\omega\left(t_{2}\right)\right)}{4},
\end{aligned}
$$

where $p$ denotes as above the right derivative of $\varphi$ on the interval $[0, \delta)$ and $\omega_{0}=\omega(t)$ for any $t \in\left(0, \gamma_{0}\right)$; note that by the definition of $\gamma_{0}$, we have $\omega_{0}-\omega\left(t_{2}\right)>0$. Hence,

$$
\begin{aligned}
\int_{0}^{\gamma_{0}} & \varphi\left(\left(x \chi_{e_{\gamma_{0}}}\right)^{*}(t)\right) \omega(t) d t+\int_{\gamma_{0}}^{t_{2}} \varphi\left(\left(x \chi_{A_{\gamma_{0}}}\right)^{*}\left(t-\gamma_{0}\right)\right) \omega(t) d t \\
& +\int_{t_{2}}^{\gamma} \varphi\left(\left(x \chi_{A_{t_{2}}}\right)^{*}\left(t-t_{2}\right)\right) \omega(t) d t \\
\leq & \int_{0}^{\gamma_{0}-a} \varphi\left(\left(x \chi_{e_{\gamma_{0}}}\right)^{*}(t)\right) \omega(t) d t+\int_{\gamma_{0}-a}^{\gamma_{0}} \varphi\left(\left(x \chi_{A_{t_{2}}}\right)^{*}\left(t-\left(\gamma_{0}-a\right)\right)\right) \omega(t) d t \\
& +\int_{\gamma_{0}}^{t_{2}} \varphi\left(\left(x \chi_{A_{\gamma_{0}}}\right)^{*}\left(t-\gamma_{0}\right)\right) \omega(t) d t \\
& +\int_{t_{2}}^{t_{2}+a} \varphi\left(\left(x \chi_{e_{\gamma_{0}}}\right)^{*}\left(t-\left(t_{2}-\gamma_{0}+a\right)\right)\right) \omega(t) d t+\int_{t_{2}+a}^{\gamma} \varphi\left(\left(x \chi_{A_{t_{2}}}\right)^{*}\left(t-t_{2}\right)\right) \omega(t) d t \\
& -\frac{3 a p u_{0}\left(\omega_{0}-\omega\left(t_{2}\right)\right)}{4} \\
\leq & \int_{0}^{\gamma} \varphi\left(x^{*}(t)\right) \omega(t) d t-\frac{3 a p u_{0}\left(\omega_{0}-\omega\left(t_{2}\right)\right)}{4}=1-\frac{3 a p u_{0}\left(\omega_{0}-\omega\left(t_{2}\right)\right)}{4} .
\end{aligned}
$$

Now assume that $\left(x \chi_{e_{\gamma_{0}}}\right)^{*}\left(\gamma_{0}-a\right)>\frac{u_{0}}{4}$. Then

$$
m\left(e_{\gamma_{0}} \cap\left(A_{x, y, u_{0}}^{+} \cup A_{x, y, u_{0}}^{-} \cup B_{x, u_{0}}\right)\right)>\gamma_{0}-a \geq \frac{3}{4} \gamma_{0},
$$

whence we get

$$
m\left(e_{\gamma_{0}} \cap B_{y, u_{0}}\right)<\frac{1}{4} \gamma_{0}
$$


Therefore, by the inequality $\max \left(m\left(A_{x, y, u_{0}}^{+}\right), m\left(A_{x, y, u_{0}}^{-}\right)\right)<t_{1} \leq \frac{1}{8} \gamma_{0}$, we obtain

$$
m\left(e_{\gamma_{0}} \cap\left(A_{x, y, u_{0}}^{+} \cup A_{x, y, u_{0}}^{-} \cup B_{y, u_{0}}\right)\right)<\frac{1}{2} \gamma_{0}<\gamma_{0}-a,
$$

and, in consequence, $\left(y \chi_{e_{0}}\right) *\left(\gamma_{0}-a\right)<\frac{u_{0}}{4}$. Simultaneously, by formulas (34) and (38) and the equality $t_{2}=\gamma_{0}+a$, we have

$$
m\left(B_{y, u_{0}} \cap A_{t_{2}}\right)>\frac{t_{0}}{2}+\frac{\gamma_{0}}{4}-\frac{\gamma_{0}}{4}-a>\frac{t_{0}}{2}-\frac{\gamma_{0}}{4}-a \geq 3 a
$$

Thus, $\left(y \chi_{t_{t_{2}}}\right)^{*}(a)>u_{0}$, which gives a possibility to repeat the investigations from (36) and (37) for $y$. In consequence, we have

$$
I_{\varphi, \omega}\left(\frac{x+y}{2}\right) \leq 1-\frac{3 a p u_{0}\left(\omega_{0}-\omega\left(t_{2}\right)\right)}{8} .
$$

Recapitulating Case 2, by inequalities (24), (30) and (39), we get inequality (20) for

$$
q=\min \left(\delta\left(\frac{\eta c}{4}\right), \delta\left(\frac{p u_{0} \omega_{0} t_{1}}{2}\right), \frac{3 a p u_{0}\left(\omega_{0}-\omega\left(t_{2}\right)\right)}{8}\right) .
$$

Case 3. Finally, assume that $\int_{0}^{\gamma} \varphi(\delta) \omega(t) d t \geq 1$ and $\gamma_{0}=0$. For arbitrary fixed $v_{\delta}>\delta$, we define the sets $A_{x, v_{\delta}}$ and $A_{y, v_{\delta}}$ by formulas (22) and (23). If $\max \left(I_{\varphi, \omega}\left(x \chi_{A_{x, v_{\delta}}}\right), I_{\varphi, \omega}\left(y \chi_{A_{y, v_{\delta}}}\right)\right) \geq$ $\frac{1}{8}$, then proceeding analogously as in Case 2 , we get inequality (24) with the constant $\delta\left(\frac{\eta}{32}\right)$. If $\max \left(I_{\varphi, \omega}\left(x \chi_{A_{x, v_{\delta}}}\right), I_{\varphi, \omega}\left(y \chi_{A_{y, \nu_{\delta}}}\right)\right)<\frac{1}{8}$, then we define $t_{0}>0$ and $u_{0}>0$ by the equalities

$$
\int_{0}^{t_{0}} \varphi\left(v_{\delta}\right) \omega(t) d t=\frac{3}{4} \quad \text { and } \quad \int_{0}^{\gamma} \varphi\left(u_{0}\right) \omega(t) d t=\frac{1}{8} .
$$

We have $t_{0}<\gamma, u_{0}<\delta$ and $\min \left(m\left(A_{x, u_{0}}\right), m\left(A_{y, u_{0}}\right)\right) \geq t_{0}$, where the sets $A_{x, u_{0}}$ and $A_{x, u_{0}}$ are defined by formulas (26) and (27). By the assumption $\gamma_{0}=0$, we can find two positive constants $t_{2}$ and $t_{3}$ such that $0<t_{3}<t_{2}<\frac{t_{0}}{2}$ and $\omega\left(t_{3}\right)>\omega\left(t_{2}\right)$. Let

$$
t_{1}=\frac{t_{3}}{8} \quad \text { and } \quad \omega_{1}=\int_{0}^{t_{1}} \omega(t) d t
$$

If $m\left(A_{x, y, u_{0}}^{+}\right) \geq t_{1}$ or $m\left(A_{x, y, u_{0}}^{-}\right) \geq t_{1}$, where the sets $A_{x, y, u_{0}}^{+}$and $A_{x, y, u_{0}}^{-}$are defined by formulas (28) and (29), then analogously as in Case 2, we obtain inequality (30) with the constant $\delta\left(\frac{p u_{0} \omega_{1}}{2}\right)$.

In the case when $\max \left(m\left(A_{x, y, u_{0}}^{+}\right), m\left(A_{x, y, u_{0}}^{-}\right)\right)<t_{1}$, we define the sets $B_{x, u_{0}}$ and $B_{y, u_{0}}$ by formulas (31) and (32). We have

$$
\min \left(m\left(B_{x, u_{0}}\right), m\left(B_{y, u_{0}}\right)\right) \geq t_{0}-2 t_{1} \geq \frac{7}{8} t_{0}
$$

Defining $a=\frac{\min \left(t_{3}, \frac{t_{0}}{2}-t_{2}\right)}{4}$ and repeating the procedure from Case 2, putting $t_{3}$ in place of $\gamma_{0}$, we get inequality (39) with the constant $\frac{3 a p u_{0}\left(\omega\left(t_{3}\right)-\omega\left(t_{2}\right)\right)}{8}$.

Summarizing Case 3, we get inequality (20) with

$$
q=\min \left(\delta\left(\frac{\eta}{32}\right), \delta\left(\frac{p u_{0} \omega_{1}}{2}\right), \frac{3 a p u_{0}\left(\omega\left(t_{3}\right)-\omega\left(t_{2}\right)\right)}{8}\right) .
$$


Theorem 2.5 Let $0<\alpha<\gamma<\infty$ and $0 \leq a_{\varphi}=\delta$. Then the Orlicz-Lorentz function space $\Lambda_{\varphi, \omega}$ is uniformly non-square if and only if $\varphi \in \Delta_{2}(\infty), \psi \in \Delta_{2}(\infty)$, $\omega$ is regular and $\alpha \in$ $\left(\frac{\gamma}{2}, \gamma\right)$.

Proof Necessity. Condition $\alpha \in\left(\frac{\gamma}{2}, \gamma\right)$ follows from Theorem 2.2, while the necessity of remaining conditions can be proved as in Theorem 2.4.

Sufficiency. Analogously as in Theorem 2.4, it is enough to show that there exists $q>0$ such that inequality $(20)$ holds for any $x, y \in S\left(\Lambda_{\varphi, \omega}\right)$.

First note that the space $\Lambda_{\varphi, \omega}([0, \alpha))$, in opposite to the space $\Lambda_{\varphi, \omega}=\Lambda_{\varphi, \omega}([0, \gamma))$, is uniformly monotone (see Theorem 1.2). Hence, by [52, Theorem 6], for all $\delta>0$ there exists $p(\delta)>0$ such that for any $u \in B\left(\Lambda_{\varphi, \omega}([0, \alpha))\right)$ and any $v \in \Lambda_{\varphi, \omega}([0, \alpha))$ with $m\{\operatorname{supp} u \cap$ $\operatorname{supp} v\}=0$ and $\|v\| \geq \delta$, we have

$$
\|u+v\| \geq(1+p(\delta))\|u\|
$$

Now, for any fixed $x, y \in\left(\Lambda_{\varphi, \omega}\right)$, we denote

$$
A_{x, y}=\left\{t \in[0, \gamma): \max \{|x(t)|,|y(t)|\}>a_{\varphi}\right\} .
$$

In order to show (20), we will consider two cases.

Case 1. If $m\left(A_{x, y}\right) \leq \alpha$, then we define

$$
\tilde{x}=x \circ \sigma \text { and } \tilde{y}=y \circ \sigma \text {, }
$$

where $\sigma:\left[0, m\left(A_{x, y}\right)\right) \rightarrow A_{x, y}$ is a measure preserving transformation (see [54, Theorem 17, p.410]). Obviously $\varphi \circ \tilde{x}, \varphi \circ \tilde{y}, \varphi \circ \frac{\tilde{x}+\tilde{y}}{2}$ and $\varphi \circ \frac{\tilde{x}-\tilde{y}}{2}$ are equimeasurable with $\varphi \circ x \chi_{A_{x, y}}$, $\varphi \circ y \chi_{A_{x, y}}, \varphi \circ \frac{x+y}{2} \chi_{A_{x, y}}$ and $\varphi \circ \frac{x+y}{2} \chi_{A_{x, y}}$, respectively. Therefore, by Theorem 2.4, there exists $q(\alpha)>0$ independent of $x$ and $y$ such that

$$
\begin{aligned}
\min \left(I_{\varphi, \omega}\left(\frac{x-y}{2}\right), I_{\varphi, \omega}\left(\frac{x+y}{2}\right)\right) & =\min \left(I_{\varphi, \omega}\left(\left(\frac{x-y}{2}\right) \chi_{A_{x, y}}\right), I_{\varphi, \omega}\left(\left(\frac{x+y}{2}\right) \chi_{A_{x, y}}\right)\right) \\
& =\min \left(I_{\varphi, \omega}\left(\frac{\widetilde{x}-\tilde{y}}{2}\right), I_{\varphi, \omega}\left(\frac{\widetilde{x}+\widetilde{y}}{2}\right)\right) \leq 1-q(\alpha) .
\end{aligned}
$$

Case 2. Let now $m\left(A_{x, y}\right)>\alpha$. Denote by $m_{0} \in \mathbb{N}$ the smallest possible number satisfying $m_{0}(\alpha-\gamma / 2) \geq \alpha$ and let $p\left(1 / 2 m_{0}\right)$ be the constant from inequality (40) for $\delta=1 / 2 m_{0}$. Fix $\varepsilon>0$ satisfying

$$
\left(1+p\left(\frac{1}{2 m_{0}}\right)\right)(1-6 \varepsilon)>1 \text { and } \varepsilon<\frac{1}{12} .
$$

Since $\psi \in \Delta_{2}(\infty)$, for $a_{\varepsilon}$ satisfying the equality

$$
\int_{0}^{\alpha} \varphi\left(a_{\varepsilon}\right) \omega(t) d t=\varepsilon
$$

analogously as in Case 1 of Theorem 2.4, we can find $\eta=\eta\left(a_{\varepsilon}\right) \in(0,1)$ such that

$$
\varphi\left(\frac{u}{2}\right)<\frac{1-\eta}{2} \varphi(u)
$$


for all $u \geq a_{\varepsilon}$. We may assume without loss of generality that

$$
\min \left(I_{\varphi, \omega}\left(\frac{x-y}{2}\right), I_{\varphi, \omega}\left(\frac{x+y}{2}\right)\right) \geq 1-\varepsilon .
$$

Applying [16, Property $7^{\circ}$, p.64], we can find sets $e_{\alpha}(+)=e_{\alpha}\left(\varphi \circ\left(\frac{x+y}{2}\right)\right)$ and $e_{\alpha}(-)=e_{\alpha}(\varphi \circ$ $\left.\left(\frac{x-y}{2}\right)\right)$ of measure $\alpha$ such that

$$
\begin{aligned}
& \int_{0}^{\alpha}\left(\varphi \circ\left(\frac{x+y}{2}\right)\right)^{*}(t) d t=\int_{e_{\alpha}(+)} \varphi \circ\left(\frac{x+y}{2}\right)(t) d t, \\
& \int_{0}^{\alpha}\left(\varphi \circ\left(\frac{x-y}{2}\right)\right)^{*}(t) d t=\int_{e_{\alpha}(-)} \varphi \circ\left(\frac{x-y}{2}\right)(t) d t .
\end{aligned}
$$

Let us define the sets

$$
\begin{aligned}
& A^{+}=\left\{t \in e_{\alpha}(+): \max (|x(t)|,|y(t)|) \geq a_{\varepsilon}\right\}, \\
& A_{1}^{+}=\left\{t \in A^{+}: x(t) y(t)>0\right\}, \\
& A_{2}^{+}=\left\{t \in A^{+}: x(t) y(t) \leq 0\right\}
\end{aligned}
$$

and

$$
\begin{aligned}
& A^{-}=\left\{t \in e_{\alpha}(-): \max (|x(t)|,|y(t)|) \geq a_{\varepsilon}\right\}, \\
& A_{1}^{-}=\left\{t \in A^{-}: x(t) y(t) \geq 0\right\}, \\
& A_{2}^{-}=\left\{t \in A^{-}: x(t) y(t)<0\right\} .
\end{aligned}
$$

From [15, Theorem 2.6, p.49] it follows that there are functions $u_{+}$and $u_{-}$both equimeasurable with $\omega \chi_{[0, \alpha]}$ and satisfying the equalities

$$
\begin{aligned}
& \int_{0}^{\alpha}\left(\varphi \circ\left(\frac{x+y}{2}\right)\right)^{*}(t) \omega(t) d t=\int_{e_{\alpha}(+)} \varphi \circ\left(\frac{x+y}{2}\right)(t) u_{+}(t) d t \\
& \int_{0}^{\alpha}\left(\varphi \circ\left(\frac{x-y}{2}\right)\right)^{*}(t) \omega(t) d t=\int_{e_{\alpha}(-)} \varphi \circ\left(\frac{x-y}{2}\right)(t) u_{-}(t) d t
\end{aligned}
$$

By the Hardy-Littlewood inequality, we have

$$
\begin{aligned}
& \int_{e_{\alpha}(+) \backslash A^{+}} \varphi \circ\left(\frac{x+y}{2}\right)(t) u_{+}(t) d t \\
& \quad \leq \int_{0}^{\alpha}\left(\varphi \circ\left(\left(\frac{x+y}{2}\right) \chi_{e_{\alpha}(+) \backslash A^{+}}\right)\right)^{*}(t)\left(u_{+} \chi_{e_{\alpha}(+) \backslash A^{+}}\right)^{*}(t) d t<\int_{0}^{\alpha} \varphi\left(a_{\varepsilon}\right) \omega(t) d t=\varepsilon,
\end{aligned}
$$

whence by (43), we conclude that

$$
\int_{A^{+}} \varphi \circ\left(\frac{x+y}{2}\right)(t) u_{+}(t) d t \geq 1-2 \varepsilon .
$$


Similarly, we get

$$
\int_{A^{-}} \varphi \circ\left(\frac{x-y}{2}\right)(t) u_{-}(t) d t \geq 1-2 \varepsilon
$$

The remaining part of the proof of Case 2 will be divided into three subcases.

Case 2.1. Suppose $\int_{A_{2}^{+}} \varphi \circ\left(\frac{x+y}{2}\right)(t) u_{+}(t) d t \geq \varepsilon$. Then

$$
\varphi\left(\left(\frac{x+y}{2}\right)(t)\right) \leq \varphi\left(\frac{\max (|x(t)|,|y(t)|)}{2}\right) \leq \frac{1-\eta}{2}\{\varphi(x(t))+\varphi(y(t))\}
$$

for $m$-a.e. $t \in A_{2}^{+}$. Hence, by equality (44), we get

$$
\begin{aligned}
I_{\varphi, \omega}\left(\frac{x+y}{2}\right)= & \int_{0}^{\alpha} \varphi\left(\left(\frac{x+y}{2}\right)^{*}(t)\right) \omega(t) d t=\int_{e_{\alpha}(+)} \varphi \circ\left(\frac{x+y}{2}\right)(t) u_{+}(t) d t \\
\leq & \frac{1}{2} \int_{e_{\alpha}(+) \backslash A_{2}^{+}}\{\varphi(x(t))+\varphi(y(t))\} u_{+}(t) d t \\
& +\frac{1-\eta}{2} \int_{A_{2}^{+}}\{\varphi(x(t))+\varphi(y(t))\} u_{+}(t) d t \\
\leq & \frac{1}{2}\left\{\int_{e_{\alpha}(+)} \varphi(x(t)) u_{+}(t) d t+\int_{e_{\alpha}(+)} \varphi(y(t)) u_{+}(t) d t\right\}-\eta \varepsilon \\
\leq & 1-\eta \varepsilon .
\end{aligned}
$$

Case 2.2. If $\int_{A_{1}^{-}} \varphi \circ\left(\frac{x-y}{2}\right)(t) u_{-}(t) d t \geq \varepsilon$, then analogously as above, we can show that

$$
I_{\varphi, \omega}\left(\frac{x-y}{2}\right) \leq 1-\eta \varepsilon
$$

Case 2.3. Finally, we will prove that the remaining case

$$
\int_{A_{2}^{+}} \varphi \circ\left(\frac{x+y}{2}\right)(t) u_{+}(t) d t<\varepsilon \quad \text { and } \quad \int_{A_{1}^{-}} \varphi \circ\left(\frac{x-y}{2}\right)(t) u_{-}(t) d t<\varepsilon
$$

is not possible. In the opposite case, by (46) and (47), we get

$$
\int_{A_{1}^{+}} \varphi \circ\left(\frac{x+y}{2}\right)(t) u_{+}(t) d t \geq 1-3 \varepsilon \quad \text { and } \quad \int_{A_{2}^{-}} \varphi \circ\left(\frac{x+y}{2}\right)(t) u_{-}(t) d t \geq 1-3 \varepsilon
$$

Since $A_{1}^{+} \cap A_{2}^{-}=\emptyset$, we can assume without loss of generality that $m\left(A_{1}^{+}\right) \leq \gamma / 2$. Moreover, by the Hardy-Littlewood inequality and convexity of the modular $I_{\varphi, \omega}$, we obtain

$$
\begin{aligned}
1-3 \varepsilon & \leq \int_{A_{1}^{+}} \varphi \circ\left(\frac{x+y}{2}\right)(t) u_{+}(t) d t \\
& \leq \int_{0}^{m\left(A_{1}^{+}\right)}\left(\varphi \circ\left(\left(\frac{x+y}{2}\right) \chi_{A_{1}^{+}}\right)\right)^{*}(t)\left(u_{+} \chi_{A_{1}^{+}}\right)^{*}(t) d t \\
& \leq \int_{0}^{m\left(A_{1}^{+}\right)}\left(\varphi \circ\left(\left(\frac{x+y}{2}\right) \chi_{A_{1}^{+}}\right)\right)^{*}(t) \omega(t) d t
\end{aligned}
$$




$$
\begin{aligned}
& =I_{\varphi, \omega}\left(\left(\frac{x+y}{2}\right) \chi_{A_{1}^{+}}\right) \leq \frac{1}{2} I_{\varphi, \omega}\left(x \chi_{A_{1}^{+}}\right)+\frac{1}{2} I_{\varphi, \omega}\left(y \chi_{A_{1}^{+}}\right) \\
& =\frac{1}{2} \int_{0}^{m\left(A_{1}^{+}\right)}\left(\varphi \circ x \chi_{A_{1}^{+}}\right)^{*}(t) \omega(t) d t+\frac{1}{2} \int_{0}^{m\left(A_{1}^{+}\right)}\left(\varphi \circ y \chi_{A_{1}^{+}}\right)^{*}(t) \omega(t) d t .
\end{aligned}
$$

Since $I_{\varphi, \omega}(x)=I_{\varphi, \omega}(y)=1$, so

$$
\int_{0}^{m\left(A_{1}^{+}\right)}\left(\varphi \circ x \chi_{A_{1}^{+}}\right)^{*}(t) \omega(t) d t \geq 1-6 \varepsilon \text { and } \int_{0}^{m\left(A_{1}^{+}\right)}\left(\varphi \circ y \chi_{A_{1}^{+}}\right)^{*}(t) \omega(t) d t \geq 1-6 \varepsilon
$$

Similarly,

$$
\int_{0}^{m\left(A_{2}^{-}\right)}\left(\varphi \circ x \chi_{A_{2}^{-}}\right)^{*}(t) \omega(t) d t \geq 1-6 \varepsilon \quad \text { and } \quad \int_{0}^{m\left(A_{2}^{-}\right)}\left(\varphi \circ y \chi_{A_{2}^{-}}\right)^{*}(t) \omega(t) d t \geq 1-6 \varepsilon
$$

Let $e_{(\alpha-\gamma / 2)}=e_{(\alpha-\gamma / 2)}\left(\varphi \circ x \chi_{A_{2}^{-}}\right) \subset A_{2}^{-}$be such that $m\left(e_{(\alpha-\gamma / 2)}\right)=\alpha-\gamma / 2$ and

$$
\int_{0}^{\alpha-\gamma / 2}\left(\varphi \circ x \chi_{A_{2}^{-}}\right)^{*}(t) d t=\int_{e_{(\alpha-\gamma / 2)}} \varphi \circ x \chi_{A_{2}^{-}}(t) d t=\int_{e_{(\alpha-\gamma / 2)}} \varphi \circ x \chi_{e_{(\alpha-\gamma / 2)}}(t) d t
$$

Then, by the definition of $m_{0}$, the first inequality in (50) and the second inequality in (41), we get

$$
\left\|\varphi \circ x \chi_{e_{(\alpha-\gamma / 2)}}\right\|_{\omega}=\int_{0}^{\alpha-\gamma / 2}\left(\varphi \circ x \chi_{A_{2}^{-}}\right)^{*}(t) \omega(t) d t \geq \frac{1-6 \varepsilon}{m_{0}} \geq \frac{1}{2 m_{0}} .
$$

Consequently, by (40) (note that $m\left(\operatorname{supp}\left(\varphi \circ x \chi_{A_{1}^{+}}+\varphi \circ x \chi_{e_{(\alpha-\gamma / 2)}}\right)\right) \leq \gamma / 2+\alpha-\gamma / 2=\alpha$ ) and first inequalities of formulas (49) and (41), we obtain

$$
\begin{aligned}
1 & =\int_{0}^{\alpha} \varphi\left(x^{*}(t)\right) \omega(t) d t \geq \int_{0}^{\alpha}\left(\varphi \circ x \chi_{A_{1}^{+}}+\varphi \circ x \chi_{e_{(\alpha-\gamma / 2)}}\right)^{*}(t) \omega(t) d t \\
& =\left\|\varphi \circ x \chi_{A_{1}^{+}}+\varphi \circ x \chi_{e_{(\alpha-\gamma / 2)}}\right\|_{\omega} \geq\left(1+p\left(\frac{1}{2 m_{0}}\right)\right)\left\|\varphi \circ x \chi_{A_{1}^{+}}\right\|_{\omega} \\
& \geq\left(1+p\left(\frac{1}{2 m_{0}}\right)\right)(1-6 \varepsilon)>1,
\end{aligned}
$$

which is a contradiction.

Summarizing both cases, we get inequality (20) with $q=\min (q(\alpha), \eta \varepsilon)$. 


\section{Author details}

${ }^{1}$ Faculty of Mathematics and Computer Science, Adam Mickiewicz University, Umultowska 87, Poznań, 61-614, Poland.

${ }^{2}$ Institute of Mathematics of Electric Faculty, Poznań University of Technology, Piotrowo 3a, Poznań, 60-965, Poland.

\section{Acknowledgements}

The authors thank the referees for valuable comments and suggestions. All authors are supported partially by the State Committee for Scientific Research, Poland, Grant No. N N201 362236.

\section{Received: 2 October 2012 Accepted: 8 January 2013 Published: 25 January 2013}

\section{References}

1. James, RC: Uniformly non-square Banach spaces. Ann. Math. 80, 542-550 (1964)

2. James, RC: Super-reflexive spaces with bases. Pac. J. Math. 41, 409-419 (1972)

3. García-Falset, J, Llorens-Fuster, E, Mazcuñan-Navarro, EM: Uniformly nonsquare Banach spaces have the fixed point property for nonexpansive mappings. J. Funct. Anal. 233, 494-514 (2006)

4. Sundaresan, K: Uniformly non-square Orlicz spaces. Nieuw Arch. Wiskd. 14, 31-39 (1966)

5. Hudzik, H: Uniformly non-(1) Orlicz spaces with Luxemburg norm. Stud. Math. 81, 271-284 (1985)

6. Hudzik, H: Locally uniformly non- $\left.\right|_{n} ^{(1)}$ Orlicz spaces. Proceedings of the 13 th Winter School on Abstract Analysis, Srni 1985. Rend. Circ. Mat. Palermo, Ser. II 10, 49-56 (1985)

7. Hudzik, H, Kamińska, A, Kurc, W: Uniformly non- $\left.\right|_{n} ^{(1)}$ Musielak-Orlicz spaces. Bull. Pol. Acad. Sci., Math. 35, 441-448 (1987)

8. Denker, M, Hudzik, H: Uniformly non- /(1) Musielak-Orlicz sequence spaces. Proc. Indian Acad. Sci. Math. Sci. 101, 71-86 (1991)

9. Cui, YA, Jie, L, Płuciennik, R: Local uniform nonsquareness in Cesaro sequence spaces. Ann. Soc. Math. Pol, 1 Comment. Math. 37, 47-58 (1997)

10. Kato, M, Maligranda, L: On James and Jordan-von Neumann constants of Lorentz sequence spaces. J. Math. Anal. Appl. 258, 457-465 (2001)

11. Maligranda, L, Petrot, N, Suantai, S: On the James constant and B-convexity of Cesàro and Cesàro-Orlicz sequence spaces. J. Math. Anal. Appl. 326, 312-331 (2007)

12. Kamińska, A, Kubiak, D: On isometric copies of $I_{\infty}$ and James constants in Cesàro-Orlicz sequence spaces. J. Math. Anal. Appl. 372, 574-584 (2010)

13. Cerdà, J, Hudzik, H, Mastyło, M: On the geometry of some Calderón-Lozanovskii interpolation spaces. Indag. Math. 6(1), 35-49 (1995)

14. Foralewski, P, Hudzik, H, Kolwicz, P: Non-squareness properties of Orlicz-Lorentz sequence spaces. J. Funct. Anal. 264, 605-629 (2013)

15. Bennett, C, Sharpley, R: Interpolation of Operators. Academic Press, New York (1988)

16. Krein, SG, Petunin, Jul, Semenov, EM: Interpolation of Linear Operators. Transl. Math. Monogr., vol. 54. Am. Math. Soc., Providence (1982)

17. Lindenstrauss, J, Tzafriri, L: Classical Banach Spaces II, Function Spaces. Springer, Berlin (1979)

18. Chilin, VI, Dodds, PG, Sedaev, AA, Sukochev, FA: Characterizations of Kadec-Klee properties in symmetric spaces of measurable functions. Trans. Am. Math. Soc. 348(12), 4895-4918 (1996)

19. Chen, ST: Geometry of Orlicz spaces. Diss. Math. 356, 1-204 (1996)

20. Maligranda, L: Orlicz Spaces and Interpolation. Seminars in Math., vol. 5. Universidade Estadual de Campinas, Campinas (1989)

21. Musielak, J: Orlicz Spaces and Modular Spaces. Lecture Notes in Math., vol. 1034. Springer, Berlin (1983)

22. Lorentz, GG: On the theory of space $\Lambda$. Pac. J. Math. 1(3), 411-429 (1951)

23. Halperin, I: Uniform convexity in function spaces. Duke Math. J. 21, 195-204 (1954)

24. Kamińska, A: Uniform convexity of generalized Lorentz spaces. Arch. Math. 56, 181-188 (1991)

25. Hudzik, H, Kamińska, A: Monotonicity properties of Lorentz spaces. Proc. Am. Math. Soc. 123(9), $2715-2721$ (1995)

26. Kamińska, A: Some remarks on Orlicz-Lorentz spaces. Math. Nachr. 147, 29-38 (1990)

27. Kamińska, A: Extreme points in Orlicz-Lorentz spaces. Arch. Math. 55, 173-180 (1990)

28. Hudzik, H, Kamińska, A, Mastyło, M: Geometric properties of some Calderón-Lozanovskiï space and Orlicz-Lorentz spaces. Houst. J. Math. 22(3), 639-663 (1996)

29. Kamińska, A, Lin, PK, Sun, H: Uniformly normal structure of Orlicz-Lorentz spaces. In: Interaction Between Functional Analysis, Harmonic Analysis, and Probability. Columbia, Missouri, 1994. Lecture Notes Pure Appl. Math., vol. 175, pp. 229-238. Dekker, New York (1996)

30. Hudzik, H, Kamińska, A, Mastyło, M: On geometric properties of Orlicz-Lorentz spaces. Can. Math. Bull. 40(3), 316-329 (1997)

31. Cerdà, J, Hudzik, H, Kamińska, A, Mastyło, M: Geometric properties of symmetric spaces with applications to Orlicz-Lorentz spaces. Positivity 2, 311-337 (1998)

32. Hudzik, H, Kamińska, A, Mastyło, M: On the dual of Orlicz-Lorentz space. Proc. Am. Math. Soc. 130(6), 1645-1654 (2002)

33. Kamińska, A, Raynaud, Y: Isomorphic PP-subspaces in Orlicz-Lorentz sequence spaces. Proc. Am. Math. Soc. 134(8), 2317-2327 (2006)

34. Levis, FE, Cuenya, HH: Gateaux differentiability in Orlicz-Lorentz spaces and applications. Math. Nachr. 280(11), 1282-1296 (2007)

35. Levis, FE, Cuenya, HH, Priori, AN: Best constant approximants in Orlicz-Lorentz spaces. Ann. Soc. Math. Pol., 1 Comment. Math. 48(1), 59-73 (2008)

36. Foralewski, P, Hudzik, H, Szymaszkiewicz, L: On some geometric and topological properties of generalized Orlicz-Lorentz sequence spaces. Math. Nachr. 281(2), 181-198 (2008)

37. Foralewski, P, Hudzik, H, Szymaszkiewicz, L: Local rotundity structure of generalized Orlicz-Lorentz sequence spaces. Nonlinear Anal. 68, 2709-2718 (2008) 
38. Kamińska, A, Raynaud, Y: Isomorphic copies in the lattice $E$ and its symmetrization $E^{(*)}$ with applications to Orlicz-Lorentz spaces. J. Funct. Anal. 257, 271-331 (2009)

39. Gong, WZ, Shi, ZR: Points of monotonicity in Orlicz-Lorentz function spaces. Nonlinear Anal. 73, 1300-1317 (2010)

40. Foralewski, P: Some fundamental geometric and topological properties of generalized Orlicz-Lorentz function spaces. Math. Nachr. 284(8-9), 1003-1023 (2011)

41. Foralewski, P: Rotundity structure of local nature for generalized Orlicz-Lorentz function spaces. Nonlinear Anal. 74, 3912-3925 (2011)

42. Foralewski, P, Hudzik, H, Kaczmarek, R, Krbec, M, Wójtowicz, M: On the moduli and characteristic of monotonicity in Orlicz-Lorentz function spaces. J. Convex Anal. 20, 4 (2013, in press)

43. Kolwicz, P: Rotundity properties in Calderón-Lozanovskil spaces. Houst. J. Math. 31(3), 883-912 (2005)

44. Foralewski, P, Kolwicz, P: Local uniform rotundity in Calderón-Lozanovskiï spaces. J. Convex Anal. 14(2), 395-412 (2007)

45. Lorentz, GG: Some new functional space. Ann. Math. 51(1), 37-55 (1950)

46. Halperin, l: Function spaces. Can. J. Math. 5, 273-288 (1953)

47. Carothers, NL, Dilworth, SJ: Equidistributed random variables in $L_{p, q}$. J. Funct. Anal. 84(1), 146-159 (1989)

48. Carothers, NL, Dilworth, SJ, Trautman, DA: On the geometry of the unit spheres of the Lorentz spaces $L_{w, 1}$. Glasg. Math. J. 34, 21-25 (1992)

49. Carothers, NL, Haydon, R, Lin, PK: On the isometries of Lorentz function spaces. Isr. J. Math. 84, $265-287$ (1993)

50. Foralewski, P: Some remarks on the geometry of Lorentz spaces $\Lambda_{1, w}$. Indag. Math. 23, 361-376 (2012)

51. Birkhoff, G: Lattice Theory. Amer. Math. Soc., Providence, RI (1967)

52. Hudzik, H, Kamińska, A, Mastyło, M: Monotonicity and rotundity properties in Banach lattices. Rocky Mt. J. Math. 30(3), 933-950 (2000)

53. Ciesielski, M, Kamińska, A, Kolwicz, P, Płuciennik, R: Monotonicity and rotundity of Lorentz spaces $\Gamma_{p, w}$. Nonlinear Anal. 75, 2713-2723 (2012)

54. Royden, HL: Real Analysis. Macmillan Publishing Company, New York (1988)

55. Chen, ST, Hudzik, H: On some convexities of Orlicz and Orlicz-Bochner spaces. Comment. Math. Univ. Carol. 29(1), $13-29(1988)$

doi:10.1186/1029-242X-2013-32

Cite this article as: Foralewski et al.: Non-squareness properties of Orlicz-Lorentz function spaces. Journal of Inequalities and Applications 2013 2013:32

\section{Submit your manuscript to a SpringerOpen ${ }^{\ominus}$ journal and benefit from:}

- Convenient online submission

- Rigorous peer review

- Immediate publication on acceptance

- Open access: articles freely available online

- High visibility within the field

- Retaining the copyright to your article 